\title{
Multi-genomic analysis of the Cation Diffusion Facilitator transporters from algae
}

Aniefon Ibuot, ${ }^{a}$ Andrew P. Dean, ${ }^{b}$ and Jon K. Pittman ${ }^{c, *}$

a Department of Science Technology, Akwa Ibom State Polytechnic, Ikot Osurua, P.M.B 1200.Ikot Ekpene, Akwa Ibom State, Nigeria

${ }^{\mathrm{b}}$ Department of Natural Sciences, Faculty of Science and Engineering, Manchester Metropolitan University, Oxford Road, Manchester, M1 5GD, UK

${ }^{c}$ Department of Earth and Environmental Sciences, School of Natural Sciences, Faculty of Science and Engineering, The University of Manchester, Michael Smith Building, Oxford Road, Manchester M13 9PT, UK

\section{${ }^{*}$ Corresponding Author:}

Dr Jon Pittman, Department of Earth and Environmental Sciences, The University of Manchester, Michael Smith Building, Oxford Road, Manchester M13 9PT, UK; Tel: +44 (0)161 275 5235; Fax: +44 (0)161 275 5082; Email: jon.pittman@manchester.ac.uk

Orchid ID: 0000-0001-7197-1494 


\begin{abstract}
Metal transport processes are relatively poorly understood in algae in comparison to higher plants and other eukaryotes. A screen of genomes from 33 taxonomically diverse algal species was conducted to identify members of the Cation Diffusion Facilitator (CDF) family of metal ion transporter. All algal genomes contained at least one CDF gene with four species having $>10$ CDF genes (median of 5 genes per genome), further confirming that this is a ubiquitous gene family. Phylogenetic analysis suggested a CDF gene organisation of five groups, which includes Zn-CDF, Fe/Zn-CDF and Mn-CDF groups, consistent with previous phylogenetic analyses, and two functionally undefined groups. One of these undefined groups was algal specific although excluded chlorophyte and rhodophyte sequences. The majority of sequences ( 22 out of 26 sequences) from this group had a putative ion binding site motif within transmembrane domain 2 and 5 that was distinct from other CDF proteins, such that alanine or serine replaced the conserved histidine residue. The phylogenetic grouping was supported by sequence cluster analysis. Yeast heterologous expression of CDF proteins from Chlamydomonas reinhardtii indicated $\mathrm{Zn}^{2+}$ and $\mathrm{Co}^{2+}$ transport function by CrMTP1, and $\mathrm{Mn}^{2+}$ transport function by CrMTP2, CrMTP3 and CrMTP4, which validated the phylogenetic prediction. However, the Mn-CDF protein CrMTP3 was also able to provide zinc and cobalt tolerance to the $\mathrm{Zn}$ - and Co-sensitive zrc1cot1 yeast strain. There is wide diversity of CDF transporters within the algae lineage, and some of these genes may be attractive targets for future applications of metal content engineering in plants or microorganisms.
\end{abstract}




\section{Introduction}

Knowledge of the transport pathways for metal influx, efflux and subcellular metal partitioning in plant and algal cells is critical in order to understand the processes of essential metal homeostasis within the cell. These will include pathways for essential trace metals such as zinc, copper and manganese, which are required for many biochemical functions including as enzyme co-factors or structural components of proteins (Andreini et al. 2008). Furthermore, both essential and non-essential metals, such as cadmium, are toxic at elevated concentrations and so transporters play a key role in protecting the cell against metal toxicity (Kramer et al. 2007). This protection can be mediated by metal efflux from the cell or intracellular metal sequestration into compartments such as the vacuole (Sharma et al. 2016). Manipulation of expression of specific transporter proteins, such as by genetic engineering, can therefore be used to enhance metal tolerance of an organism (Antosiewicz et al. 2014). Moreover, manipulation of metal sequestration may also provide increased total metal content within a cell whilst still providing metal tolerance, and would be useful for metal bioremediation applications (Cheng et al. 2019; Ibuot et al. 2017).

Algae are one such group of organisms that have been proposed as potentially suitable for metal bioremediation (Suresh Kumar et al. 2015), but if genetic engineering of metal transporters is to be tested in algae then molecular targets that have been functionally characterised are needed. Furthermore, knowledge of the gene families that encode metal transporters across taxonomically diverse algal species provides understanding of the evolution of metal homeostasis within these important photosynthetic organisms. There is a long history of the study of metal homeostasis and transport processes in algae during the second half of the last century (particularly for green microalgae species and brown seaweeds), including physiological investigation of metal micronutrient requirements and metal toxicity responses, and biochemical characterisation of metal transport (Fig. S1). However, the molecular identification of individual metal transporters and genomic scale analysis of whole gene families has only begun in the last fifteen years since the availability 
of algal genome sequences. For example, genomic analyses of green microalgae, such as Chlamydomonas reinhardtii, and red microalgae, such as Cyanidioschyzon merolae indicate that there are a wide variety of metal transporter pathways that are conserved between microalgae and higher plants alongside other eukaryotes such as yeast (Hanikenne et al. 2005; Blaby-Haas and Merchant 2012). The identification of putative algal metal transporter genes has been complemented by other genome-scale analyses such as by proteomic and transcriptomic methods (Malasarn et al. 2013; Beauvais-Flück et al. 2019; Puente-Sánchez et al. 2018; Hsieh et al. 2013; Khatiwada et al. 2020), and subsequent functional characterisation of individual metal transporters (Ibuot et al. 2017; Tsednee et al. 2019; Chang et al. 2020).

One important metal transporter family is the Cation Diffusion Facilitator (CDF) family, which are referred to as Metal Transport Proteins (MTP) in higher plants (Kolaj-Robin et al. 2015; Ricachenevsky et al. 2013). CDF-type transporters have been reported to facilitate the transport of metals including cadmium (Cd), cobalt (Co), copper (Cu), iron (Fe), manganese $(\mathrm{Mn})$, nickel $(\mathrm{Ni})$, and zinc $(\mathrm{Zn})$, and are found virtually in all organisms covering the Eukaryote, Eubacteria and Archaea kingdoms (Cubillas et al. 2013; Nies and Silver 1995). In yeast and plants, CDF/MTP proteins localise to membranes of various intracellular compartments, in particular the vacuolar membrane but also the mitochondria, endoplasmic reticulum (ER), Golgi/trans-Golgi and pre-vacuolar compartment, where they are involved in the efflux of metals from the cytoplasm into these organelles (Haney et al. 2005;

Ricachenevsky et al. 2013). For example, in Saccharomyces cerevisiae, CDF proteins mediate $\mathrm{Zn}^{2+}$ and $\mathrm{Co}^{2+}$ transfer into the vacuole (ScZrc1 and ScCot1), $\mathrm{Zn}^{2+}$ transfer into the ER (ScZrg17 and ScMsc2) and Fe ${ }^{2+}$ transfer from the mitochondria (ScMmt1 and ScMmt2) (Ellis et al. 2005; Li et al. 2014; MacDiarmid et al. 2002). In Arabidopsis thaliana, AtMTP1 and related orthologues including AtMTP3 play a major role in the storage and sequestration of $\mathrm{Zn}^{2+}$ in the vacuole (Arrivault et al. 2006; Kobae et al. 2004), AtMTP5 and AtMTP12 transport $\mathrm{Zn}^{2+}$ into the Golgi (Fujiwara et al. 2015), while AtMTP8 and AtMTP11 mediate 
$\mathrm{Mn}^{2+}$ transport into the vacuole and Golgi (Delhaize et al. 2007; Peiter et al. 2007; Eroglu et al. 2016).

These main metal substrates $\left(\mathrm{Zn}^{2+}, \mathrm{Co}^{2+}, \mathrm{Mn}^{2+}, \mathrm{Fe}^{2+}\right)$ of the higher plant and fungal CDF family proteins are all essential micronutrients but can cause major toxicity if they accumulate at excess concentration. $\mathrm{Zn}$ is an important co-factor for many enzymes, such as carbonic anhydrase that is required for photosynthetic carbon fixation, and is associated with many Zn-finger containing transcription factors, and has a structural role with some proteins (Decaria et al. 2010; Hänsch and Mendel 2009). Co is an essential metal in a few organisms, such as where it is required for vitamin $B_{12}$ and used by coenzyme $B_{12^{-}}$ dependent enzymes (Renfrew et al. 2018). Mn also has many essential enzyme co-factor requirements, such as $\mathrm{Mn-dependent} \mathrm{superoxide} \mathrm{dismutase} \mathrm{and} \mathrm{the} \mathrm{water} \mathrm{splitting} \mathrm{oxygen}$ evolution process of Photosystem II (Pittman 2005; Socha and Guerinot 2014). Finally, Fe also plays many co-factor roles and protein structural roles, and is essential for electron transport processes (Hänsch and Mendel 2009).

The current understanding of CDF/MTP proteins in algae is limited. The CDF gene family appears to be present in all previously examined algal species but specific details about CDF family structures within individual species are unknown (Blaby-Haas and Merchant 2012, 2017; Gustin et al. 2011). Phylogenetic analysis will be able to reveal some useful details since substrate specificity seems to be related to phylogeny (Cubillas et al. 2013; Montanini et al. 2007). Within the well-studied model microalga C. reinhardtii there are five putative MTP genes (CrMTP1 to CrMTP5). Some of these genes are transcriptionally induced by Zn or Mn deficiency conditions (Allen et al. 2007; Malasarn et al. 2013), but to date only CrMTP4 has been functionally characterised as a $\mathrm{Mn}^{2+}$ and $\mathrm{Cd}^{2+}$ transporter (Ibuot et al. 2017). This was determined by performing over-expression of CrMTP4 in C. reinhardtii yielding enhanced $\mathrm{Cd}$ tolerance, and by using yeast ( $\mathrm{S}$. cerevisiae) heterologous expression where CrMTP4 was able to rescue the Mn sensitive phenotype of the pmr1 yeast mutant. In this study, we aimed to perform a large-scale phylogenetic analysis of putative MTP genes from available algal genome sequences in order to evaluate the potential diversity within this 
broad taxonomic lineage. Furthermore, a characterisation of additional members of the $C$. reinhardtii MTP gene family was carried out by using yeast heterologous expression to infer the metal substrates and determine metal tolerance functions.

\section{Materials and Methods}

\section{Bioinformatic analysis}

Thirty three algal species genomes were screened from one Charophyta: Klebsormidium nitens; 13 Chlorophyta: Bathycoccus prasinos RCC1105, Botryococcus braunii, Chlamydomonas reinhardtii, Chlorella sp. NC64A, Chromochloris zofingiensis, Coccomyxa subellipsoidea C-169, Dunaliella salina, Micromonas pusilla CCMP1545, Micromonas sp. RCC299, Ostreococcus lucimarinus, Ostreococcus sp. RCC809, Ostreococcus tauri RCC4221, Volvox carteri; two Pelagophyta: Aureococcus anophagefferens clone 1984, Pelagophyceae sp. CCMP2097; two Cryptophyta: Cryptophyceae sp. CCMP2293, Guillardia theta CCMP2712; three Rhodophyta: Cyanidioschyzon merolae, Galdieria sulphuraria 074W, Porphyra umbilicalis; four Haptophyta: Emiliania huxleyi CCMP1516, Pavlovales sp. CCMP2436, Phaeocystis antarctica CCMP1374, Phaeocystis globosa Pg-G; four Bacillariophyta: Fragilariopsis cylindrus CCMP 1102, Phaeodactylum tricornutum CCAP 1055/1, Pseudo-nitzschia multiseries CLN-47, Thalassiosira pseudonana CCMP 1335; three Ochrophyta: Ectocarpus siliculosus Ec 32, Nannochloropsis oceanica CCMP1779, Ochromonadaceae sp. CCMP2298; and one Chlorarachniophyta: Bigelowiella natans CCMP2755. In addition, other species genomes were screened for comparison from the cyanobacterium Nostoc punctiforme PCC 73102, human Homo sapiens, land plant Arabidopsis thaliana, yeast Saccharomyces cerevisiae, and the thraustochytrid Schizochytrium aggregatum ATCC 28209. Finally sequences from ten bacteria and one archeae were used. Genome details and individual ID numbers or accession numbers for the sequences used are provided in Supplementary Information and Table S1. 
Genomes were screened by BLAST using C. reinhardtii and S. cerevisiae CDF protein sequences. In addition, genomes were screened for the presence of the Pfam domain PF01545. All sequences were then manually examined by sequence alignment to remove any false positive sequences that had been mis-annotated. Amino acid multiple sequence alignments were performed using Clustal Omega (Madeira et al. 2019). For visual analysis sequence alignments were shaded using BoxShade v.3.21 and manually annotated. Prediction of transmembrane domains (TMD) was performed by hydropathy analysis using TMHMM v.2.0 (Krogh et al. 2001), and was performed by consensus topology prediction using TOPCONS (Tsirigos et al. 2015). His-rich loop presence was determined by manual analysis by identifying the presence of three or more histidine residues within the putative cytosolic loop region between TMD 4 and 5. Conserved residues within TMD2 and TMD5 of Group 1 (Zn-CDF) and Group 5 sequences were identified using WebLogo v.2.8.2 (Crooks et al. 2004).

Phylogenetic relationships at the amino acid level were performed using full length sequences, essentially as described previously (Emery et al. 2012; Pittman and Hirschi 2016). Sequence alignment outputs from Clustal Omega were used to generate maximum likelihood phylogenies under the WAG+F model of amino acid substitution with $\Gamma$-distributed rates across sites, as implemented in RAxML v.7.1, with tree confidence determined from 1000 replications using the fast bootstrap method (Stamatakis 2006; Stamatakis et al. 2008). The trees were viewed using FigTree v.1.3 (http://tree.bio.ed.ac.uk/software/figtree).

The relationship between sequences was further quantified using Cluster Analysis of Sequences (CLANS) (Frickey and Lupas 2004), which was used to conduct an all-against-all BLAST of unaligned sequences. Pairwise similarities between protein sequences were displayed in a 2-dimensional cluster plot using CLANS Jar from the MPI Bioinformatics Toolkit (Zimmermann et al. 2018). The $P$ value was set to $1 \times 10^{-5}$ and the analysis was run for 50,000 iterations. 


\section{Microalgae cultivation and RNA isolation}

C. reinhardtii (CC125) was obtained from the Chlamydomonas Resource Center, USA, and was grown photo-heterotrophically in batch culture in Tris-acetate-phosphate (TAP) medium buffered at pH 7.0, as described previously (Harris 1989), in $200 \mathrm{~mL}$ glass flasks on an orbital shaker rotating at $2 \mathrm{~Hz}$, at $25^{\circ} \mathrm{C}$ under cool-white fluorescent lights $\left(150 \mu \mathrm{mol} \mathrm{m}^{-2}\right.$ $\mathrm{s}^{-1}$ ) with a 16-h:8-h light:dark regime. Cultures were inoculated with the same starting cell density as determined by cell counting to give an initial cell count of $\sim 65 \times 10^{3}$ cells $\mathrm{mL}^{-1}$. RNA was isolated from exponential growing C. reinhardtii CC125 cells using Trizol reagent (Life Technologies) and further purified by phenol/chloroform extraction and precipitation with isopropanol.

\section{MTP full length cDNA cloning}

C. reinhardtii genomic sequence and gene model information was obtained from Phytozome v.12 using v.5.5 of the C. reinhardtii genome annotations and gene-specific oligonucleotide primers incorporating restriction enzyme sites (Table S2) were designed to amplify the fulllength CrMTP1, CrMTP2, and CrMTP3 cDNA sequences by RT-PCR from C. reinhardtii CC125 RNA. First-strand cDNA was generated from $1 \mu \mathrm{g}$ of DNase-treated RNA using Superscript III reverse transcriptase (Life Technologies) and an oligo(dT) primer, then KAPA HiFi DNA polymerase (Kapa Biosystems) and gene-specific primers were used for each PCR amplification, with an annealing temperature of $60^{\circ} \mathrm{C}$ and 35 amplification cycles. Following amplification, the PCR products were cloned into pGEM-T Easy plasmid (Promega) for propagation and sequencing (GATC Biotech) to confirm sequence fidelity. The yeast expression plasmid piUGpd was used to allow expression of each cDNA under the control of the constitutive yeast GAPDH promoter and selection of the URA3 gene (Nathan et al. 1999). CrMTP1 cDNA was sub-cloned into the Xbal and Sacl sites of piUGpd, while CrMTP2 and CrMTP3 cDNA were sub-cloned into the BamHI and Xbal sites of piUGpd. CrMTP4 in piUGpd was generated previously (Ibuot et al. 2017). 


\section{Quantitative gene expression analysis}

C. reinhardtii CC125 was cultured in TAP medium for three days then cells were harvested and frozen in liquid $\mathrm{N}_{2}$. RNA was isolated and cDNA was produced as described above. Expression for each MTP gene was determined by quantitative real-time PCR (qPCR) using short amplicon primers that were designed to be specific to each gene (Table S3). For qPCR a SYBR Green core qPCR kit (Eurogentec) and a StepOnePlus machine (ThermoFisher) using the SYBR Green detection program was used. Transcript abundance for each MTP was normalised to CBLP transcript abundance. Reactions were run in triplicate and qPCR analysis was performed as described previously (Bajhaiya et al. 2016).

\section{Yeast heterologous expression and metal tolerance assays}

Yeast (S. cerevisiae) strains pmr1 (MATa; his $3 \Delta 1$; leu2 $\Delta 0$; met15 $\Delta 0$; ura3 $\Delta 0$; pmr1::kanMX4) (Euroscarf, Frankfurt, Germany), zrc1 cot1 (MATa; his3 $\Delta 1$; leu2 $\Delta 0$; met15 $\Delta$; ura3 $\Delta 0$; zrc1::natMX; $\operatorname{cot1}:: k a n M X 4)$ (Drager et al. 2004) (kindly provided by Ute Krämer) and the corresponding wild type strain BY4741 (MATa; his3 $\Delta 1$; leu2 $\Delta 0$; met15 $\Delta 0$; ura3 $\Delta 0)$ (Euroscarf) were each transformed with each MTP-piUGpd plasmid or empty piUGpd plasmid using the lithium acetate-polyethylene glycol method. Colonies were selected by growth at $30^{\circ} \mathrm{C}$ in synthetic defined medium minus uracil (SD -Ura) as described previously (Pittman et al. 2004). Expression of each MTP cDNA in yeast was confirmed by RT-PCR using the appropriate MTP qPCR primer pair (Table S3) and RNA extracted from yeast using Trizol reagent, then RT-PCR was performed as described above. PCR products were examined on a $1 \%$ agarose gel stained with SafeView (NBS Biologicals).

Metal tolerance assays were performed essentially as described previously (Pittman et al. 2009), on solid SD -Ura medium with or without $2 \mathrm{mM} \mathrm{ZnCl}, 5 \mathrm{mM} \mathrm{MnCl}_{2}$ or $0.8 \mathrm{mM}$ $\mathrm{CoCl}_{2}$ metal salts. These concentrations were chosen as the most appropriate to restrict growth of the $\mathrm{Zn}$ - and Co-sensitive zrt1 cot1 strain and the Mn-sensitive pmr1 strain but still allow the wild type BY4741 to grow. For growth in liquid SD -Ura medium, yeast strains were grown in medium with or without $10 \mathrm{mM}$ or $20 \mathrm{mM} \mathrm{CoCl}, \mathrm{MnCl}_{2}$, or $\mathrm{ZnCl}_{2}$ metal salts. 
Cell density was determined by absorbance measurement at $600 \mathrm{~nm}$ while yeast biomass was determined by weighing a cell pellet from a set volume of culture after it was dried at $60^{\circ} \mathrm{C}$ for $24 \mathrm{~h}$. Internal metal content in yeast grown in liquid SD -Ura medium containing 20 $\mathrm{mM} \mathrm{ZnCl} 2$ or $20 \mathrm{mM} \mathrm{MnCl}_{2}$, respectively, was determined by inductively coupled plasma atomic emission spectroscopy (ICP-AES) essentially as described previously using a PerkinElmer Optima 5300 (Webster et al. 2011). It was estimated using Visual Minteq v.3.0 metal speciation modelling that the free ion concentrations available to the cells was $10.4 \mathrm{mM} \mathrm{Z \textrm {n } ^ { 2 + }}$ and $12.6 \mathrm{mM} \mathrm{Mn}^{2+}$. Cells were centrifuged for $10 \mathrm{~min}$ at $3000 \mathrm{~g}$ then resuspended in $10 \mathrm{~mL}$ of $10 \mathrm{mM}$ EDTA for $5 \mathrm{~min}$, then re-centrifuged and washed with $15 \mathrm{~mL}$ Milli-Q water. Cell pellets were dried at $60{ }^{\circ} \mathrm{C}$ for $24 \mathrm{~h}$ and then digested in $0.5 \mathrm{~mL}$ of ultrapure concentrated nitric acid at $70{ }^{\circ} \mathrm{C}$ for $2 \mathrm{~h}$. Samples were diluted in Milli-Q water to $2 \%(\mathrm{v} / \mathrm{v})$ concentration of acid and analysed by ICP-AES. All samples were calibrated using a matrix-matched serial dilution of Specpure multi-element plasma standard solution 4 (Alfa Aesar) set by linear regression.

\section{Results}

\section{Phylogenetic analysis of algal CDF protein sequences}

Previous phylogenetic analyses of CDF sequences are inconsistent in terms of clade structure and nomenclature of groups, with proposed numbers of CDF sub-groups ranging from 3 to 18 groups (Migeon et al. 2010; Cubillas et al. 2013; Gustin et al. 2011; Montanini et al. 2007). Montanini et al. (2007) divided the CDF family into three major groups based on substrate specificity, referred to as Zn-CDF, Fe/Zn-CDF and Mn-CDF, while Gustin et al. (2011) further sub-divided the higher plant CDF members into seven groups. Cubillas et al. (2013) identified 18 CDF groups that were differentiated mostly depending on whether they contained eukaryote, bacteria or archaea derived sequences. Here we performed a phylogenetic analysis that focussed on available algal genomes, including from one charophyte, 13 chlorophytes, two pelagophytes, two cryptophytes, three rhodophytes, four 
haptophytes, four bacillariophytes, three ochrophytes and one chlorarachniophyte (Table 1). A representative 'algal-like' thraustochytrid (S. aggregatum) was also included. First, each genome was analysed by a combination of BLAST and Pfam signature searching to collate CDF sequences. Each algal genome examined had at least one CDF sequence and the numbers of CDF transporter genes ranged from one in the red alga G. sulphuraria to 14 genes in the haptophyte bloom microalga $P$. antarctica (Table 1). The exact numbers of CDF genes within the more recently annotated genomes should be considered with caution since incorrect annotation and genome assembly errors can lead to genes being missed.

To generate the phylogenetic tree, the algal sequences were aligned with representative sequences from bacteria (Escherichia coli, Bacillus subtilis, Bradyrhizobium diazoefficiens, Cupriavidus metallidurans, Rhizobium etli, Sinorhizobium meliloti, Streptococcus pneumoniae, Thermus thermophiles), archaea (Methanosarcina acetivorans), cyanobacteria ( $N$. punctiforme), animals (H. sapiens), fungi (S. cerevisiae), and land plants (A. thaliana), which had all been used in previous phylogenetic analyses and in most cases have known substrate specificity (Table S1). The constructed phylogenetic tree contained 226 sequences, including 15 prokaryotic, 10 human, 12 plant, 6 yeast, 7 thraustochytrid and 176 algal sequences, of which 42 were chlorophyte sequences. Despite the dominance of many new algal sequences, the tree structure was consistent with previous CDF trees (Gustin et al. 2011; Montanini et al. 2007). It possessed distinct groups containing many of the known $\mathrm{Zn}^{2+}, \mathrm{Fe}^{2+}$ and $\mathrm{Mn}^{2+}$ transporting CDF proteins characterised in human, yeast, $A$. thaliana and bacteria, which were recognisable as the previously named Zn-CDF, Fe/ZnCDF and Mn-CDF groups (Fig. 1). Group 1 (Zn-CDF) could have been considered as two or three separate groups, including a bacterial-specific clade (shown in dark blue in Fig. 1) and two eukaryotic groups (an AtMTP1 - AtMTP4 containing group, and an AtMTP5 and AtMTP12 containing group), which all include CDF proteins with experimentally confirmed $\mathrm{Zn}^{2+}$ transport activity (e.g. Arrivault et al. 2006; Fujiwara et al. 2015; Chao and Fu 2004; Liuzzi and Cousins 2004; Kobae et al. 2004; MacDiarmid et al. 2002), indicating functional conservation across the whole Group 1. All taxonomic classes of algae possessed a Group 
$1 \mathrm{Zn}-\mathrm{CDF}$, apart from the Chlorarachniophyta B. natans, although 5 of the 13 chlorophytes, including all three Ostreococcus spp. appear to lack a Zn-CDF (Table 1).

Group 2 (Fe/Zn-CDF), which includes $\mathrm{Fe}^{2+}$ and/or $\mathrm{Zn}^{2+}$ transporting proteins such as yeast ScMMT1 and ScMMT2, and C. metallidurans CmFieF, is rich in algal CDF sequences, including a large number of haptophyte, bacillariophyte, and ochrophyte sequences, but includes only two chlorophyte and no rhodophyte sequences (Fig. 1). A predominantly bacterial sub-group also containing five algal sequences (shown in light green in Fig. 1) might be considered as a separate group since it contains CDF proteins with known $\mathrm{Mn}^{2+}$, $\mathrm{Ni}^{2+}$ and $\mathrm{Co}^{2+}$ transport activities (Cubillas et al. 2013), rather than $\mathrm{Fe}^{2+} / \mathrm{Zn}^{2+}$. Group 3 and Group 4 (Mn-CDF) are considered as two separate groups rather than a single group. Group 4 contains many known plant $\mathrm{Mn}^{2+}$ transporting CDFs, such as AtMTP11 (Delhaize et al. 2007; Peiter et al. 2007), and is also enriched in chlorophyte and haptophyte sequences. Group 3 is also rich in sequences from all chlorophyte species, and includes CDF proteins such as AtMTP7 and HsZnt9 that have no currently confirmed substrate (Fig. 1). Although indirect evidence indicates $\mathrm{Zn}^{2+}$ transport activity by HsZnt9 (Perez et al. 2017), Group 3 is referred to as a separate undefined CDF group. Finally, the tree displays a fifth algal-specific group, which is also referred to as undefined. Group 5 sequences are missing in all of the examined higher plant, charophyte and chlorophyte genomes (Fig. S2).

In order to provide validation for the group structure proposed for the CDF tree shown in Fig. 1, a phenetic sequence clustering method was performed using CLANS (Frickey and Lupas 2004). Nearly all of the sequences fell into five clusters, which completely matched the Groups 1 to 5 determined by the phylogenetic tree (Fig. 2). In particular, the analysis fully validated the separation of Group 3 and 4 into independent groups, which were both very tightly clustered. The Group 2 sequences were fairly tightly clustered and indicated partly that this group should not be further divided (shown by light green and dark green symbols overlapping in Fig. 2), although six of the sequences within this sub-group, including five algal proteins from Pelagophyceae sp., T. pseudonana, E. siliculosus and Ochromonadaceae sp., and the bacterial Sm_0683 protein from S. meliloti 
did not cluster with the other Group 2 sequences, and are instead classified as 'unassigned' (Table 1). Group 1 and 5 sequences also formed clearly distinct clusters although these were not as tightly clustered as the other groups, and there was some overlap between four of the Group 5 sequences with Group 1. Overall, this CLANS analysis validated the fivegroup organisation for these CDF sequences.

\section{Structural features of $\mathrm{Zn}-\mathrm{CDF}$ related sequences from algae}

Conserved amino acid residues within TMD2 and 5 have been demonstrated to be critical for metal ion binding, in particular, two residues from TMD2 and two from TMD5 form a metal binding site referred to as 'binding site A' (Kolaj-Robin et al. 2015; Barber-Zucker et al. 2017). The presence of residues HxxxD-HxxxD within the TMD2 and TMD5 regions, respectively, was conserved for the algal-dominated Group 1 proteins examined here (Fig. 3), which is consistent with previous analysis of Zn-CDF proteins (Montanini et al. 2007). In contrast, the Group 5 proteins displayed a binding site A motif that was predominantly A/SxxxD-HxxxD, such that Ala or Ser replaced the conserved His (Fig. 3). Only 4 of the 26 Group 5 proteins had the HxxxD-HxxxD motif that was highly conserved within Group 1 proteins. Indeed, these were the four Group 5 proteins that partially overlapped with the Group 1 cluster (Fig. 2).

Most of the $\mathrm{Zn}^{2+}$-transporting CDF proteins within Group 1 possess a His-rich cytosolic loop between TMD4 and 5, and this loop may play a role in metal selectivity, as some $\mathrm{Zn}-\mathrm{CDF}$ transporters are specific for $\mathrm{Zn}^{2+}$ while others can also transport metals including $\mathrm{Co}^{2+}$ (Kawachi et al. 2012; Podar et al. 2012). However, the presence of a His-rich sequence is not universal, and while most the Zn-CDF proteins examined here possess an obvious His-rich loop region, including most of the chlorophyte and bacillariophyte sequences, a few do not, including some of the human Znt proteins, A. thaliana AtMTP5, and AtMTP5-related algal sequences (Fig. 4a). Another notable feature of many of the algal $\mathrm{Zn}-\mathrm{CDF}$ sequences is that the His-rich cytosolic loop is often significantly longer than the equivalent loop region present within CDF proteins such as from Arabidopsis, human and 
yeast (Fig. 4b). For example, the CrMTP1 His-rich loop is over 300 amino acids in length while the AtMTP1 loop just over 50 amino acids in length (Fig. S3). However, some algal CDF proteins, such as from Micromonas sp. and G. theta have very short His-stretch regions of less than 10 amino acids (Fig. 4b).

\section{Functional characterisation of C. reinhardtii CDF proteins}

C. reinhardtii has five CDF proteins, CrMTP1 within Group 1 (Zn-CDF), CrMTP2, CrMTP3 and CrMTP4, within Group 4 (Mn-CDF), and CrMTP5 within the undefined Group 3 (Fig. S2). To determine whether all genes are expressed within the organism, RT-PCR was performed using RNA extracted from exponential growth stage cells grown under standard conditions. Transcripts were detected for all five genes with CrMTP1, CrMTP2 and CrMTP5 showing an equivalent level of expression (between approximately $0.2-0.3 \%$ of $C B L P$ expression), while CrMTP3 and CrMTP4 had low but detectable levels of expression that was significantly reduced $(P<0.05)$ by approximately $10-20$-fold compared to that of CrMTP1, CrMTP2 and CrMTP5 (Fig. 5). Growth of C. reinhardtii in excess concentrations of metals such as $\mathrm{Cd}, \mathrm{Co}, \mathrm{Fe}, \mathrm{Mn}$ and $\mathrm{Zn}$ had no significant effect on the expression level of any of the genes (data not shown) indicating that none of these CrMTP genes are transcriptionally regulated by excess metal status.

CrMTP4 has previously been confirmed to have $\mathrm{Mn}^{2+}$ transport activity and the ability to transport $\mathrm{Cd}^{2+}$ (Ibuot et al. 2017), but the substrate specificity of the other C. reinhardtii CDF proteins is unknown. The full-length cDNA for each gene was functionally characterised by heterologous expression in yeast (Fig. 6), however, the expression of CrMTP5 was unsuccessful and therefore no functional information could be obtained for this protein. In contrast, CrMTP1, CrMTP2, CrMTP3 and CrMTP4 were all successfully expressed in a Znand Co-sensitive yeast strain zrc1 cot1 (lacking the yeast vacuolar $\mathrm{Zn}^{2+}$ and $\mathrm{Co}^{2+}$ transporters ScZRC1 and ScCOT1, respectively), and a Mn-sensitive yeast strain pmr1 (lacking the $\mathrm{Mn}^{2+}$ ATPase transporter ScPMR1) (Fig. 6b). All cDNAs were functional as indicated by their ability to suppress metal sensitive phenotypes (Fig. 6a). CrMTP4 was used 
as a positive control as a confirmed Mn-CDF, and as seen with other higher plant Mn-CDF proteins, it was unable to rescue the growth of zrc1 cot1 yeast on Zn or Co media. In contrast, CrMTP1 could strongly suppress both the $\mathrm{Zn}$ and Co sensitivity of $\operatorname{zrc} 1 \cot 1$ but was unable to suppress the Mn sensitivity of pmr1, indicating that CrMTP1 is a $\mathrm{Zn}^{2+}$ and $\mathrm{Co}^{2+}$ transporter. CrMTP2 showed the identical characteristics of CrMTP4, indicating that it is a specific $\mathrm{Mn}^{2+}$ transporter (Fig. 6a). CrMTP3 was interesting as it could strongly suppress the Mn sensitivity of pmr1 but could also partially suppress the $\mathrm{Zn}$ and Co sensitivity of $z r c 1$ cot1, although not as strongly as CrMTP1. This suggested that CrMTP3 has broad substrate specificity for all three divalent cations tested. To date, none of the Mn-CDF proteins have been found to have $\mathrm{Zn}^{2+}$ or $\mathrm{Co}^{2+}$ transport activity, and appear to be largely $\mathrm{Mn}^{2+}$-specific (Delhaize et al. 2007; Montanini et al. 2007; Peiter et al. 2007).

To further confirm the $\mathrm{Zn}$, Co and Mn tolerance abilities of these proteins all cDNAs were expressed in a wild type yeast strain (BY4741) (Fig. 6b). Growth of the yeast in liquid medium was quantified in the presence of external metal addition, at concentrations that induced toxicity by inhibiting growth of wild type yeast and were relevant to metal contaminated environments (Wuana and Okieimen 2011). CrMTP1 but not CrMTP3 was able to moderately but significantly increase tolerance to $\mathrm{Zn}$ in the yeast strain as determined by cell density measurement (Fig. 7b) and by yeast biomass (Fig. 8a). However, there was no significant increase in yeast growth for any of the MTP-expressing strains in response to Co addition (Fig. 7c and Fig. 8b). All three CDF proteins (CrMTP2, CrMTP3, CrMTP4) that were able to strongly suppress the Mn sensitivity of $p m r 1$ yeast could also provide strong growth tolerance to BY4741 yeast in the presence of $20 \mathrm{mM} \mathrm{Mn} \mathrm{(Fig.} 7 \mathrm{~d}$ and Fig. 8c).

\section{Metal accumulation into yeast expressing C. reinhardtii CDF proteins}

To determine whether the expression of algal CDF proteins in yeast can provide increased metal transport into the cell, $\mathrm{Zn}$ and $\mathrm{Mn}$ concentration in yeast cell biomass was quantified by ICP-AES following growth in liquid media with added $\mathrm{Zn}$ or Mn. Prior to measurement, 
cells were washed in an EDTA solution to remove cell wall bound metals so that only internalised metals were measured. There was no significant increase in $\mathrm{Zn}$ content for any of the strains including for the CrMTP1 expressing yeast (Fig. 9a), whereas the yeast expressing CrMTP2, CrMTP3 and CrMTP4 showed an approximately 2-fold increase in Mn content within the cells (Fig. 9b), indicating that the Mn tolerance of these strains was potentially due to internal sequestration of $\mathrm{Mn}$.

\section{Discussion}

\section{Conserved phylogenetic structure of algal CDF transporters}

CDF metal transporters are a ubiquitous family of proteins across life, and this analysis confirms their presence across the wide range of taxonomically and physiologically diverse photosynthetic organisms that are collectively referred to as algae. CDF transporters appear to be principally involved in the transfer of various essential metal ions including $\mathrm{Zn}^{2+}, \mathrm{Mn}^{2+}$, $\mathrm{Fe}^{2+}$ and $\mathrm{Co}^{2+}$ into internal organelles either for delivery to specific subcellular-localised proteins that require metal co-factors or for providing metal tolerance through internal sequestration (Kolaj-Robin et al. 2015; Ricachenevsky et al. 2013). Although other classes of primary or secondary energised metal transporters can play similar roles (Blaby-Haas and Merchant 2012), CDF transporters are clearly indispensable in algae since each algal genome that has been analysed here has at least one CDF isoform. Furthermore, the algaldominated phylogenetic analysis performed in this study was consistent with a previously determined CDF phylogenetic structure, whereby a small number of major groups can be differentiated on the basis of $\mathrm{Zn}^{2+}, \mathrm{Fe}^{2+} / \mathrm{Zn}^{2+}$ and $\mathrm{Mn}^{2+}$ substrate specificity, plus undefined substrate specificity (Montanini et al. 2007). The fact that this phylogenetic structure is conserved in many algal species suggests that it was determined early in the evolution of photosynthetic organisms. In particular, the Zn-CDF and Mn-CDF groups are the most conserved across the algal genomes examined here. Nevertheless, some algal species have lost particular CDF types; for example, all three Ostreococcus spp. lack Group 1 (Zn- 
CDF) and Group 2 (Fe/Zn-CDF) genes, and in fact most chlorophytes lack Group 2 (Fe/Zn-

CDF) genes, while all three red algae examined (C. merolae, G. sulphuraria and $P$. umbilicalis) lack Group 2 (Fe/Zn-CDF) and the undefined Group 3 genes. The functional relevance of this is unclear but may indicate that functional redundancy with other transporter classes (e.g. $\mathrm{Zn}^{2+}$ or $\mathrm{Mn}^{2+}$ P-type ATPases; Fe ${ }^{2+}$ or $\mathrm{Mn}^{2+}$ transporting VIT-like proteins) is not essential and such redundancy has therefore not been selected in many of these organisms.

\section{A novel algal-specific phylogenetic group with distinct ion binding site motif}

We have also proposed the presence of a fifth phylogenetic group (Group 5) that is most closely related to Group 1 (Zn-CDF) but appears to be distinct. This group is almost exclusively algal-specific apart from a $S$. aggregatum isoform, but includes no chlorophyte or rhodophyte sequences. The difference in sequence for the TMD2 half of the metal binding site A motif of the majority of the Group 5 proteins further demonstrates a divergence with the other groups. This TMD2 motif is typically HxxxD or DxxxD in most CDF proteins, such as in the $\mathrm{Zn}^{2+}$-transporting AtMTP1 and CrMTP1 (HxxxD), and in the $\mathrm{Mn}^{2+}$-transporting AtMTP11 and CrMTP4 (DxxxD), but the Group 5 TMD2 motif was A/SxxxD. Mutation of the His residue within this motif to Ala in the AtMTP1 Zn-CDF protein, the PtdMTP1 Zn-CDF protein from Populus trichocarpa $x$ deltoides and the RmCzcD Zn-CDF protein from Ralstonia metallidurans all caused loss of function (Blaudez et al. 2003; Kawachi et al. 2012; Anton et al. 2004), perhaps indicating that these Group 5 proteins would not be functional for $\mathrm{Zn}^{2+}$ transport. However, there are exceptions to these motifs where His or Asp in the first position is not essential, such as NxxxD within TMD2 of the $\mathrm{Mn}^{2+}$ transporting SpMntA from S. pneumoniae or FxxxD within TMD2 of the $\mathrm{Zn}^{2+}$ transporting ScZRG17 from S. cerevisiae (Barber-Zucker et al. 2017). Furthermore, the recently identified $\mathrm{Na}^{+}$-transporting MceT protein from the halophilic bacterium Planococcus dechangensis, which has been proposed to be a member of a novel Na-CDF clade, has a distinct YxxxS motif (Xu et al. 2019). 
Currently we are unable to predict the substrate specificity of these Group 5 algae proteins from sequence comparisons therefore future experimental analysis is needed.

\section{C. reinhardtii MTP transporters have $\mathrm{Zn}-\mathrm{CDF}$ and Mn-CDF functions}

This study has identified C. reinhardtii members of the CDF family of metal efflux transporters as $\mathrm{Zn}^{2+}, \mathrm{Co}^{2+}$ and $\mathrm{Mn}^{2+}$ transporters, and validated the function of an algal $\mathrm{Zn}$ CDF protein for the first time. CrMTP1 clearly groups in the Group 1 (Zn-CDF) clade while CrMTP2, CrMTP3 and CrMTP4 are clearly within the Group 4 (Mn-CDF) clade, and the yeast expression experiments validated these predicted activities, as we previously observed for CrMTP4 (Ibuot et al. 2017). An intriguing exception was the apparent $\mathrm{Mn}^{2+}$ and $\mathrm{Zn}^{2+}$ transport activity by CrMTP3, the first example we are aware of for a $\mathrm{Mn}^{2+}$-transporting CDF also being linked to $\mathrm{Zn}^{2+}$ transport. A Mn-CDF from A. thaliana (AtMTP8) may transport both $\mathrm{Mn}^{2+}$ and $\mathrm{Fe}^{2+}$ (Chu et al. 2017), while the Group 1 (Zn-CDF) member HsZnt10 can transport $\mathrm{Mn}^{2+}$ (Nishito et al. 2016). While there is some sequence variation between CrMTP3 and CrMTP2/CrMTP4 that could explain this difference, future biochemical and mutagenesis studies are required to experimental validate this further.

CrMTP1 was able to efficiently provide $\mathrm{Zn}$ tolerance to yeast, indicating $\mathrm{Zn}^{2+}$ transport activity, despite the structure of CrMTP1 being distinct from previously characterised Zn-CDF proteins such as AtMTP1 and ScZRC1 due to the significantly longer His-rich loop domain between TMD4 and 5. This seems to be a structural feature for many of the algal Zn-CDF proteins. The His-rich loop has been proposed to play a role in metal ion specificity and affinity, such that shortening of the AtMTP1 loop, which also reduces the number of His residues, alters the kinetics of $\mathrm{Zn}^{2+}$ transport, while the shortened loop or specific point-mutations within the loop can allow $\mathrm{Co}^{2+}$ transport, which is otherwise poor in native AtMTP1 (Kawachi et al. 2008; Podar et al. 2012). Therefore we might predict that the longer His-loop of CrMTP1 may provide different $\mathrm{Zn}^{2+}$ and $\mathrm{Co}^{2+}$ transport kinetics compared to some of the higher plant isoforms. 
CrMTP2 and CrMTP4 but not the other C. reinhardtii CDF family genes have been shown to be transcriptionally induced by Mn deficiency, thus linking them further to $\mathrm{Mn}$ homeostasis roles (Allen et al. 2007), while CrMTP1 is induced by Zn deficiency (Malasarn et al. 2013), although we could find no evidence by RT-PCR that any of these genes are transcriptionally regulated by metal excess. CrMTP1 was able to efficiently rescue the $\mathrm{Zn}$ sensitive phenotype of the yeast vacuolar $\mathrm{Zn}^{2+} / \mathrm{H}^{+} \mathrm{CDF}$ transporter ScZRC1 and provide $\mathrm{Zn}$ tolerance in wild type yeast, thus it is proposed to provide a pathway of vacuolar $\mathrm{Zn}^{2+}$ sequestration equivalent to AtMTP1 and ScZRC1 (Kobae et al. 2004; MacDiarmid et al. 2002). Likewise, the significant Mn tolerance gained by CrMTP2, CrMTP3 or CrMTP4 that was coincident with increased Mn content within the cell is consistent with sequestration of $\mathrm{Mn}^{2+}$ into an internal compartment, possibly a vacuolar structure. C. reinhardtii lacks a large central vacuole as in typical plant cells but possess multiple lysosomal/vacuolar-like structures that are also referred to as acidocalcisomes (Blaby-Haas and Merchant 2014; Komine et al. 2000). These are acidic structures that accumulate polyphosphate and metals including $\mathrm{Ca}, \mathrm{Mg}$ and $\mathrm{Zn}$, and possess proton pumps that would be able to energise $\mathrm{H}^{+}-$ coupled $\mathrm{Zn}^{2+}$ or $\mathrm{Mn}^{2+}$ transport by an MTP protein (Ruiz et al. 2001). The identification of the red algal MTP1 orthologue from C. merolae (Cmer_CMF058C) from an acidocalcisome proteomic study (Yagisawa et al. 2009), further strengthens the likely function of algal ZnCDF proteins in vacuolar $\mathrm{Zn}^{2+}$ sequestration.

\section{Conclusions}

Knowledge of sub-cellular metal transport processes, such as by members of the CDF transporter family, not only provides improved understanding of fundamental mechanisms of metal homeostasis and metal tolerance within a cell for a given organism, but may provide tools for biotechnological approaches to manipulate metal content within a whole organism, tissue, or sub-cellular compartment. Previous studies have proposed applications of CDF transporters for enhancing mineral biofortification of plants or for providing toxic metal bioremediation in plants or algae (Ibuot et al. 2017; Ricachenevsky et al. 2013; Das et al. 
2016). This present study has indicated the wide diversity of CDF transporters that exist within the algae lineage. Although the vast majority of these proteins are still uncharacterized and the potential metal substrates are unknown, it is quite possible that some of these proteins will have characteristics that are distinct to those CDF transporters already known in plants and other eukaryotes. Therefore algal CDF genes may be attractive gene targets for future genetic engineering approaches in plants or microorganisms for metal transport engineering.

\section{Acknowledgements}

This work was supported by a Government of Nigeria TETFUND PhD studentship awarded to Al. We thank Paul Lythgoe (Manchester Analytical Geochemical Unit, Department of Earth and Environmental Sciences, University of Manchester) for ICP-AES analysis. We thank Ute Krämer for providing the $\operatorname{zrc1} \cot 1$ yeast strain.

\section{Conflict of interest}

There are no conflicts to declare.

\section{References}

Allen MD, Kropat J, Tottey S, Del Campo JA, Merchant SS (2007) Manganese deficiency in Chlamydomonas results in loss of photosystem II and MnSOD function, sensitivity to peroxides, and secondary phosphorus and iron deficiency. Plant Physiol 143 (1):263-277. doi:10.1104/pp.106.088609

Andreini C, Bertini I, Cavallaro G, Holliday GL, Thornton JM (2008) Metal ions in biological catalysis: from enzyme databases to general principles. J Biol Inorg Chem 13 (8):12051218. doi:10.1007/s00775-008-0404-5

Anton A, Weltrowski A, Haney CJ, Franke S, Grass G, Rensing C, Nies DH (2004) Characteristics of zinc transport by two bacterial Cation Diffusion Facilitators from 
Ralstonia metallidurans CH34 and Escherichia coli. J Bacteriol 186 (22):7499-7507. doi:10.1128/jb.186.22.7499-7507.2004

Antosiewicz DM, Barabasz A, Siemianowski O (2014) Phenotypic and molecular consequences of overexpression of metal-homeostasis genes. Front Plant Sci 5:80. doi:10.3389/fpls.2014.00080

Arrivault S, Senger T, Kramer U (2006) The Arabidopsis metal tolerance protein AtMTP3 maintains metal homeostasis by mediating $\mathrm{Zn}$ exclusion from the shoot under Fe deficiency and Zn oversupply. Plant J 46 (5):861-879. doi:10.1111/j.1365$313 \times .2006 .02746 . x$

Bajhaiya AK, Dean AP, Zeef LAH, Webster RE, Pittman JK (2016) PSR1 is a global transcriptional regulator of phosphorus deficiency responses and carbon storage metabolism in Chlamydomonas reinhardtii. Plant Physiol 170 (3):1216-1234. doi:10.1104/pp.15.01907

Barber-Zucker S, Shaanan B, Zarivach R (2017) Transition metal binding selectivity in proteins and its correlation with the phylogenomic classification of the cation diffusion facilitator protein family. Sci Rep 7 (1):16381. doi:10.1038/s41598-017-16777-5

Beauvais-Flück R, Slaveykova VI, Cosio C (2019) Comparative study of Cu uptake and early transcriptome responses in the green microalga Chlamydomonas reinhardtii and the macrophyte Elodea nuttallii. Environ Pollut 250:331-337.

doi:10.1016/j.envpol.2019.04.032

Blaby-Haas CE, Merchant SS (2012) The ins and outs of algal metal transport. Biochim Biophys Acta 1823 (9):1531-1552. doi:10.1016/j.bbamcr.2012.04.010

Blaby-Haas CE, Merchant SS (2014) Lysosome-related organelles as mediators of metal homeostasis. J Biol Chem 289 (41):28129-28136. doi:10.1074/jbc.R114.592618

Blaby-Haas CE, Merchant SS (2017) Regulating cellular trace metal economy in algae. Curr Opin Plant Biol 39:88-96. doi:10.1016/j.pbi.2017.06.005 
Blaudez D, Kohler A, Martin F, Sanders D, Chalot M (2003) Poplar metal tolerance protein 1 confers zinc tolerance and is an oligomeric vacuolar zinc transporter with an essential leucine zipper motif. Plant Cell 15 (12):2911-2928

Chang P, Yin H, Imanaka T, Igarashi Y, Li N, Luo F (2020) The metal transporter CrNRAMP1 is involved in zinc and cobalt transports in Chlamydomonas reinhardtii. Biochem Biophys Res Commun 523 (4):880-886. doi:10.1016/j.bbrc.2019.12.121 Chao Y, Fu D (2004) Kinetic study of the antiport mechanism of an Escherichia coli zinc transporter, ZitB. J Biol Chem 279 (13):12043-12050. doi:10.1074/jbc.M313510200

Cheng SY, Show P-L, Lau BF, Chang J-S, Ling TC (2019) New prospects for modified algae in heavy metal adsorption. Trends Biotechnol 37 (11):1255-1268.

doi:10.1016/j.tibtech.2019.04.007

Chu H-H, Car S, Socha AL, Hindt MN, Punshon T, Guerinot ML (2017) The Arabidopsis MTP8 transporter determines the localization of manganese and iron in seeds. Scientific Reports 7 (1):11024. doi:10.1038/s41598-017-11250-9

Crooks GE, Hon G, Chandonia J-M, Brenner SE (2004) WebLogo: a sequence logo generator. Genome Res 14 (6):1188-1190. doi:10.1101/gr.849004

Cubillas C, Vinuesa P, Tabche ML, García-de los Santos A (2013) Phylogenomic analysis of Cation Diffusion Facilitator proteins uncovers $\mathrm{Ni}^{2+} / \mathrm{Co}^{2+}$ transporters. Metallomics 5 (12):1634-1643. doi:10.1039/C3MT00204G

Das N, Bhattacharya S, Maiti MK (2016) Enhanced cadmium accumulation and tolerance in transgenic tobacco overexpressing rice metal tolerance protein gene OsMTP1 is promising for phytoremediation. Plant Physiol Biochem 105:297-309. doi:10.1016/j.plaphy.2016.04.049

Decaria L, Bertini I, Williams RJP (2010) Zinc proteomes, phylogenetics and evolution. Metallomics 2 (10):706-709. doi:10.1039/COMT00024H

Delhaize E, Gruber BD, Pittman JK, White RG, Leung H, Miao YS, Jiang LW, Ryan PR, Richardson AE (2007) A role for the AtMTP11 gene of Arabidopsis in manganese transport and tolerance. Plant J 51 (2):198-210 
Drager DB, Desbrosses-Fonrouge AG, Krach C, Chardonnens AN, Meyer RC, SaumitouLaprade P, Kramer U (2004) Two genes encoding Arabidopsis halleri MTP1 metal transport proteins co-segregate with zinc tolerance and account for high MTP1 transcript levels. Plant J 39 (3):425-439. doi:10.1111/j.1365-313X.2004.02143.x

Ellis CD, MacDiarmid CW, Eide DJ (2005) Heteromeric protein complexes mediate zinc transport into the secretory pathway of eukaryotic cells. J Biol Chem 280 (31):2881128818. doi:10.1074/jbc.M505500200

Emery L, Whelan S, Hirschi KD, Pittman JK (2012) Protein phylogenetic analysis of $\mathrm{Ca}^{2+} /$ cation antiporters and insights into their evolution in plants. Front Plant Sci 3:1

Eroglu S, Meier B, von Wirén N, Peiter E (2016) The vacuolar manganese transporter MTP8 determines tolerance to iron deficiency-induced chlorosis in Arabidopsis. Plant Physiol 170 (2):1030-1045. doi:10.1104/pp.15.01194

Frickey T, Lupas A (2004) CLANS: a Java application for visualizing protein families based on pairwise similarity. Bioinformatics 20 (18):3702-3704.

doi:10.1093/bioinformatics/bth444

Fujiwara T, Kawachi M, Sato Y, Mori H, Kutsuna N, Hasezawa S, Maeshima M (2015) A high molecular mass zinc transporter MTP12 forms a functional heteromeric complex with MTP5 in the Golgi in Arabidopsis thaliana. FEBS J 282 (10):1965-1979. doi:10.1111/febs.13252

Gustin JL, Zanis MJ, Salt DE (2011) Structure and evolution of the plant cation diffusion facilitator family of ion transporters. BMC Evol Biol 11 (1):76. doi:10.1186/1471-2148-1176

Haney CJ, Grass G, Franke S, Rensing C (2005) New developments in the understanding of the cation diffusion facilitator family. J Ind Microbiol Biotechnol 32 (6):215-226. doi:10.1007/s10295-005-0224-3

Hanikenne M, Kramer U, Demoulin V, Baurain D (2005) A comparative inventory of metal transporters in the green alga Chlamydomonas reinhardtii and the red alga Cyanidioschizon merolae. Plant Physiol 137 (2):428-446. doi:10.1104/pp.104.054189 
Hänsch R, Mendel RR (2009) Physiological functions of mineral micronutrients $(\mathrm{Cu}, \mathrm{Zn}, \mathrm{Mn}$,

Fe, Ni, Mo, B, Cl). Curr Opin Plant Biol 12 (3):259-266. doi:10.1016/j.pbi.2009.05.006

Harris EH (1989) The Chlamydomonas Sourcebook. Academic Press, San Diego

Hsieh SI, Castruita M, Malasarn D, Urzica E, Erde J, Page MD, Yamasaki H, Casero D,

Pellegrini M, Merchant SS, Loo JA (2013) The proteome of copper, iron, zinc, and manganese micronutrient deficiency in Chlamydomonas reinhardtii. Mol Cell Proteomics 12 (1):65-86. doi:10.1074/mcp.M112.021840

Ibuot A, Dean AP, McIntosh OA, Pittman JK (2017) Metal bioremediation by CrMTP4 overexpressing Chlamydomonas reinhardtii in comparison to natural wastewater-tolerant microalgae strains. Algal Res 24:89-96. doi:10.1016/j.algal.2017.03.002

Kawachi M, Kobae Y, Kogawa S, Mimura T, Kraemer U, Maeshima M (2012) Amino acid screening based on structural modeling identifies critical residues for the function, ion selectivity and structure of Arabidopsis MTP1. FEBS J 279 (13):2339-2356. doi:10.1111/j.1742-4658.2012.08613.x

Kawachi M, Kobae Y, Mimura T, Maeshima M (2008) Deletion of a histidine-rich loop of

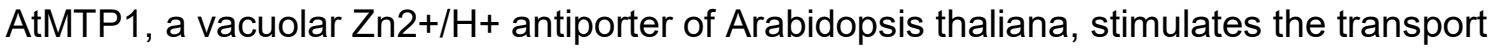
activity. J Biol Chem 283 (13):8374-8383. doi:10.1074/jbc.M707646200

Khatiwada B, Hasan MT, Sun A, Kamath KS, Mirzaei M, Sunna A, Nevalainen H (2020) Proteomic response of Euglena gracilis to heavy metal exposure - Identification of key proteins involved in heavy metal tolerance and accumulation. Algal Res 45:101764. doi:10.1016/j.algal.2019.101764

Kobae Y, Uemura T, Sato MH, Ohnishi M, Mimura T, Nakagawa T, Maeshima M (2004) Zinc transporter of Arabidopsis thaliana AtMTP1 is localized to vacuolar membranes and implicated in zinc homeostasis. Plant Cell Physiol 45 (12):1749-1758.

doi:10.1093/pcp/pci015

Kolaj-Robin O, Russell D, Hayes KA, Pembroke JT, Soulimane T (2015) Cation Diffusion Facilitator family: Structure and function. FEBS Lett 589 (12):1283-1295.

doi:10.1016/j.febslet.2015.04.007 
Komine Y, Eggink LL, Park HS, Hoober JK (2000) Vacuolar granules in Chlamydomonas reinhardtii: polyphosphate and a 70-kDa polypeptide as major components. Planta 210 (6):897-905

Kramer U, Talke IN, Hanikenne M (2007) Transition metal transport. FEBS Lett 581 (12):2263-2272. doi:10.1016/j.febslet.2007.04.010

Krogh A, Larsson B, von Heijne G, Sonnhammer ELL (2001) Predicting transmembrane protein topology with a hidden Markov model: Application to complete genomes. J Mol Biol 305 (3):567-580. doi:10.1006/jmbi.2000.4315

Li L, Miao R, Jia X, Ward DM, Kaplan J (2014) Expression of the yeast Cation Diffusion Facilitators Mmt1 and Mmt2 affects mitochondrial and cellular iron homeostasis: evidence for mitochondrial iron export. J Biol Chem 289 (24):17132-17141. doi:10.1074/jbc.M114.574723

Liuzzi JP, Cousins RJ (2004) Mammalian zinc transporters. Annu Rev Nutr 24 (1):151-172. doi:10.1146/annurev.nutr.24.012003.132402

MacDiarmid CW, Milanick MA, Eide DJ (2002) Biochemical properties of vacuolar zinc transport systems of Saccharomyces cerevisiae. J Biol Chem 277 (42):39187-39194 Madeira F, Park Ym, Lee J, Buso N, Gur T, Madhusoodanan N, Basutkar P, Tivey ARN, Potter SC, Finn RD, Lopez R (2019) The EMBL-EBI search and sequence analysis tools APIs in 2019. Nucleic Acids Res 47 (W1):W636-W641. doi:10.1093/nar/gkz268 Malasarn D, Kropat J, Hsieh SI, Finazzi G, Casero D, Loo JA, Pellegrini M, Wollman F-A, Merchant SS (2013) Zinc deficiency impacts $\mathrm{CO}_{2}$ assimilation and disrupts copper homeostasis in Chlamydomonas reinhardtii. J Biol Chem 288 (15):10672-10683. doi:10.1074/jbc.M113.455105

Migeon A, Blaudez D, Wilkins O, Montanini B, Campbell MM, Richaud P, Thomine S, Chalot M (2010) Genome-wide analysis of plant metal transporters, with an emphasis on poplar. Cell Mol Life Sci 67 (22):3763-3784. doi:10.1007/s00018-010-0445-0

Montanini B, Blaudez D, Jeandroz S, Sanders D, Chalot M (2007) Phylogenetic and functional analysis of the Cation Diffusion Facilitator (CDF) family: improved signature 
and prediction of substrate specificity. BMC Genomics 8 (1):107. doi:10.1186/1471-21648-107

Nathan DF, Vos MH, Lindquist S (1999) Identification of SSF1, CNS1, and HCH1 as multicopy suppressors of a Saccharomyces cerevisiae Hsp90 loss-of-function mutation. Proc Natl Acad Sci USA 96 (4):1409-1414

Nies DH, Silver S (1995) Ion efflux systems involved in bacterial metal resistances. J Ind Microbiol 14 (2):186-199. doi:10.1007/bf01569902

Nishito Y, Tsuji N, Fujishiro H, Takeda T-a, Yamazaki T, Teranishi F, Okazaki F, Matsunaga A, Tuschl K, Rao R, Kono S, Miyajima H, Narita H, Himeno S, Kambe T (2016) Direct comparison of manganese detoxification/efflux proteins and molecular characterization of ZnT10 protein as a manganese transporter. J Biol Chem 291 (28):14773-14787. doi:10.1074/jbc.M116.728014

Peiter E, Montanini B, Gobert A, Pedas P, Husted S, Maathuis FJM, Blaudez D, Chalot M, Sanders D (2007) A secretory pathway-localized cation diffusion facilitator confers plant manganese tolerance. Proc Natl Acad Sci USA 104 (20):8532-8537

Perez Y, Shorer Z, Liani-Leibson K, Chabosseau P, Kadir R, Volodarsky M, Halperin D, Barber-Zucker S, Shalev H, Schreiber R, Gradstein L, Gurevich E, Zarivach R, Rutter GA, Landau D, Birk OS (2017) SLC30A9 mutation affecting intracellular zinc homeostasis causes a novel cerebro-renal syndrome. Brain 140 (4):928-939. doi:10.1093/brain/awx013

Pittman JK (2005) Managing the manganese: molecular mechanisms of manganese transport and homeostasis. New Phytol 167 (3):733-742

Pittman JK, Cheng NH, Shigaki T, Kunta M, Hirschi KD (2004) Functional dependence on calcineurin by variants of the Saccharomyces cerevisiae vacuolar $\mathrm{Ca}^{2+} / \mathrm{H}^{+}$exchanger Vcx1p. Mol Microbiol 54 (4):1104-1116

Pittman JK, Edmond C, Sunderland PA, Bray CM (2009) A cation-regulated and proton gradient-dependent cation transporter from Chlamydomonas reinhardtii has a role in 
calcium and sodium homeostasis. J Biol Chem 284 (1):525-533.

doi:10.1074/jbc.M807173200

Pittman JK, Hirschi KD (2016) Phylogenetic analysis and protein structure modelling identifies distinct $\mathrm{Ca}^{2+} /$ Cation antiporters and conservation of gene family structure within Arabidopsis and rice species. Rice 9 (1):3. doi:10.1186/s12284-016-0075-8

Podar D, Scherer J, Noordally Z, Herzyk P, Nies D, Sanders D (2012) Metal selectivity determinants in a family of transition metal transporters. J Biol Chem 287 (5):3185-3196. doi:10.1074/jbc.M111.305649

Puente-Sánchez F, Díaz S, Penacho V, Aguilera A, Olsson S (2018) Basis of genetic adaptation to heavy metal stress in the acidophilic green alga Chlamydomonas acidophila. Aquat Toxicol 200:62-72. doi:10.1016/j.aquatox.2018.04.020

Renfrew AK, O'Neill ES, Hambley TW, New EJ (2018) Harnessing the properties of cobalt coordination complexes for biological application. Coord Chem Rev 375:221-233. doi:10.1016/j.ccr.2017.11.027

Ricachenevsky F, Menguer P, Sperotto R, Williams L, Fett J (2013) Roles of plant metal tolerance proteins (MTP) in metal storage and potential use in biofortification strategies. Front Plant Sci 4:144. doi:10.3389/fpls.2013.00144

Ruiz FA, Marchesini N, Seufferheld M, Govindjee, Docampo R (2001) The polyphosphate bodies of Chlamydomonas reinhardtii possess a proton-pumping pyrophosphatase and are similar to acidocalcisomes. J Biol Chem 276 (49):46196-46203

Sharma SS, Dietz K-J, Mimura T (2016) Vacuolar compartmentalization as indispensable component of heavy metal detoxification in plants. Plant, Cell Environ 39 (5):1112-1126. doi:10.1111/pce.12706

Socha AL, Guerinot ML (2014) Mn-euvering manganese: the role of transporter gene family members in manganese uptake and mobilization in plants. Front Plant Sci 5:106. doi:10.3389/fpls.2014.00106 
Stamatakis A (2006) RAxML-VI-HPC: Maximum likelihood-based phylogenetic analyses with thousands of taxa and mixed models. Bioinformatics 22 (21):2688-2690.

doi:10.1093/bioinformatics/btl446

Stamatakis A, Hoover P, Rougemont J (2008) A rapid bootstrap algorithm for the RAxML web servers. Syst Biol 57 (5):758-771. doi:10.1080/10635150802429642

Suresh Kumar K, Dahms H-U, Won E-J, Lee J-S, Shin K-H (2015) Microalgae - A promising tool for heavy metal remediation. Ecotoxicol Environ Saf 113:329-352.

doi:10.1016/j.ecoenv.2014.12.019

Tsednee M, Castruita M, Salomé PA, Sharma A, Lewis BE, Schmollinger SR, Strenkert D, Holbrook K, Otegui MS, Khatua K, Das S, Datta A, Chen S, Ramon C, Ralle M, Weber PK, Stemmler TL, Pett-Ridge J, Hoffman BM, Merchant SS (2019) Manganese colocalizes with calcium and phosphorus in Chlamydomonas acidocalcisomes and is mobilized in manganese-deficient conditions. J Biol Chem 294 (46):17626-17641. doi:10.1074/jbc.RA119.009130

Tsirigos KD, Peters C, Shu N, Käll L, Elofsson A (2015) The TOPCONS web server for consensus prediction of membrane protein topology and signal peptides. Nucleic Acids Res 43 (W1):W401-W407. doi:10.1093/nar/gkv485

Webster RE, Dean AP, Pittman JK (2011) Cadmium exposure and phosphorus limitation increases metal content in the freshwater alga Chlamydomonas reinhardtii. Environ Sci Technol 45 (17):7489-7496. doi:10.1021/es200814c

Wuana RA, Okieimen FE (2011) Heavy metals in contaminated soils: a review of sources, chemistry, risks and best available strategies for remediation. ISRN Ecology 2011:402647

Xu T, Chen H, Li J, Hong S, Shao L, Zheng X, Zou Q, Wang Y, Guo S, Jiang J (2019) Implications for cation selectivity and evolution by a novel Cation Diffusion Facilitator family member from the moderate halophile Planococcus dechangensis. Front Microbiol 10:607. doi:10.3389/fmicb.2019.00607

Yagisawa F, Nishida K, Yoshida M, Ohnuma M, Shimada T, Fujiwara T, Yoshida Y, Misumi O, Kuroiwa H, Kuroiwa T (2009) Identification of novel proteins in isolated polyphosphate 
vacuoles in the primitive red alga Cyanidioschyzon merolae. Plant J 60 (5):882-893.

doi:10.1111/j.1365-313X.2009.04008.x

Zimmermann L, Stephens A, Nam S-Z, Rau D, Kübler J, Lozajic M, Gabler F, Söding J,

Lupas AN, Alva V (2018) A completely reimplemented MPI Bioinformatics Toolkit with a new HHpred server at its core. J Mol Biol 430 (15):2237-2243.

doi:10.1016/j.jmb.2017.12.007 
Table 1. Total number and distribution of CDF transporter genes from each analysed genome into five phylogenetic groups: Group 1 (Zn-CDF), Group 2 (Fe/Zn-CDF), Group 3 (undefined CDF), Group 4 (Mn-CDF), Group 5 (undefined CDF). Gene numbers were determined from the phylogenetic analysis (Fig. 1) and supported by the cluster analysis (Fig. 2). Six sequences were classed as unassigned (UA) including five sequences where the evidence for Group 2 assignment was not supported by cluster analysis.

\begin{tabular}{|c|c|c|c|c|c|c|c|c|}
\hline & ecies & $\begin{array}{l}\text { G.1 } \\
(\mathrm{Zn})\end{array}$ & $\begin{array}{c}\text { G.2 } \\
(\mathrm{Fe} / \mathrm{Zn})\end{array}$ & $\begin{array}{c}\text { G.3 } \\
\text { (n.d.) }\end{array}$ & $\begin{array}{c}\text { G.4 } \\
(\mathrm{Mn})\end{array}$ & $\begin{array}{c}\text { G.5 } \\
\text { (n.d.) }\end{array}$ & UA & Total \\
\hline Cyanobacteria & Nostoc punctiforme & 0 & 2 & 2 & 0 & 0 & 0 & 4 \\
\hline Metazoa & Homo sapiens & 9 & 0 & 1 & 0 & 0 & 0 & 10 \\
\hline Ascomycota & $\begin{array}{l}\text { Saccharomyces } \\
\text { cerevisiae }\end{array}$ & 3 & 2 & 0 & 0 & 0 & 1 & 6 \\
\hline Embryophyta & Arabidopsis thaliana & 6 & 1 & 1 & 4 & 0 & 0 & 12 \\
\hline Thraustochytrid & $\begin{array}{l}\text { Schizochytrium } \\
\text { aggregatum }\end{array}$ & 3 & 1 & 1 & 1 & 1 & 0 & 7 \\
\hline Charophyta & Klebsormidium nitens & 4 & 1 & 1 & 2 & 0 & 0 & 8 \\
\hline \multirow[t]{13}{*}{ Chlorophyta } & Bathycoccus prasinos & 0 & 1 & 1 & 1 & 0 & 0 & 3 \\
\hline & Botryococcus braunii & 1 & 0 & 1 & 0 & 0 & 0 & 2 \\
\hline & $\begin{array}{l}\text { Chlamydomonas } \\
\text { reinhardtii }\end{array}$ & 1 & 0 & 1 & 3 & 0 & 0 & 5 \\
\hline & Chlorella sp. NC64A & 1 & 0 & 1 & 1 & 0 & 0 & 3 \\
\hline & $\begin{array}{l}\text { Chromochloris } \\
\text { zofingiensis }\end{array}$ & 1 & 0 & 1 & 2 & 0 & 0 & 4 \\
\hline & $\begin{array}{l}\text { Coccomyxa } \\
\text { subellipsoidea }\end{array}$ & 2 & 0 & 1 & 2 & 0 & 0 & 5 \\
\hline & Dunaliella salina & 2 & 0 & 1 & 1 & 0 & 0 & 4 \\
\hline & Micromonas pusilla & 0 & 0 & 1 & 1 & 0 & 0 & 2 \\
\hline & Micromonas sp. & 2 & 1 & 1 & 1 & 0 & 0 & 5 \\
\hline & $\begin{array}{l}\text { Ostreococcus } \\
\text { lucimarinus }\end{array}$ & 0 & 0 & 1 & 1 & 0 & 0 & 2 \\
\hline & Ostreococcus sp. & 0 & 0 & 1 & 1 & 0 & 0 & 2 \\
\hline & Ostreococcus tauri & 0 & 0 & 1 & 1 & 0 & 0 & 2 \\
\hline & Volvox carteri & 1 & 0 & 1 & 1 & 0 & 0 & 3 \\
\hline \multirow[t]{2}{*}{ Pelagophyta } & $\begin{array}{l}\text { Aureococcus } \\
\text { anophagefferens }\end{array}$ & 1 & 2 & 0 & 1 & 0 & 0 & 4 \\
\hline & Pelagophyceae sp. & 1 & 2 & 1 & 2 & 4 & 1 & 11 \\
\hline \multirow[t]{2}{*}{ Cryptophyta } & Cryptophyceae sp. & 1 & 1 & 0 & 0 & 1 & 0 & 3 \\
\hline & Guillardia theta & 6 & 1 & 1 & 1 & 1 & 0 & 10 \\
\hline \multirow[t]{3}{*}{ Rhodophyta } & $\begin{array}{l}\text { Cyanidioschyzon } \\
\text { merolae }\end{array}$ & 2 & 0 & 0 & 1 & 0 & 0 & 3 \\
\hline & Galdieria sulphuraria & 1 & 0 & 0 & 0 & 0 & 0 & 1 \\
\hline & Porphyra umbilicalis & 3 & 0 & 0 & 1 & 0 & 0 & 4 \\
\hline \multirow[t]{4}{*}{ Haptophyta } & Emiliania huxleyi & 3 & 4 & 0 & 1 & 3 & 0 & 11 \\
\hline & Pavlovales sp. & 2 & 2 & 0 & 2 & 3 & 0 & 9 \\
\hline & Phaeocystis antarctica & 5 & 7 & 1 & 1 & 0 & 0 & 14 \\
\hline & Phaeocystis globosa & 2 & 4 & 0 & 1 & 2 & 0 & 9 \\
\hline \multirow[t]{4}{*}{ Bacillariophyta } & Fragilariopsis cylindrus & 1 & 1 & 1 & 0 & 3 & 0 & 6 \\
\hline & $\begin{array}{l}\text { Phaeodactylum } \\
\text { tricornutum }\end{array}$ & 1 & 1 & 1 & 1 & 1 & 0 & 5 \\
\hline & $\begin{array}{l}\text { Pseudo-nitzschia } \\
\text { multiseries }\end{array}$ & 2 & 1 & 1 & 1 & 1 & 0 & 6 \\
\hline & $\begin{array}{l}\text { Thalassiosira } \\
\text { pseudonana }\end{array}$ & 1 & 1 & 1 & 1 & 0 & 1 & 5 \\
\hline \multirow[t]{3}{*}{ Ochrophyta } & Ectocarpus siliculosus & 1 & 1 & 1 & 0 & 1 & 1 & 5 \\
\hline & $\begin{array}{l}\text { Nannochloropsis } \\
\text { oceanica }\end{array}$ & 3 & 1 & 1 & 1 & 0 & 0 & 6 \\
\hline & Ochromonadaceae sp. & 1 & 2 & 0 & 0 & 2 & 2 & 7 \\
\hline Chlorarachniophyta & Bigelowiella natans & 0 & 2 & 1 & 1 & 3 & 0 & 7 \\
\hline
\end{tabular}




\section{Figures}

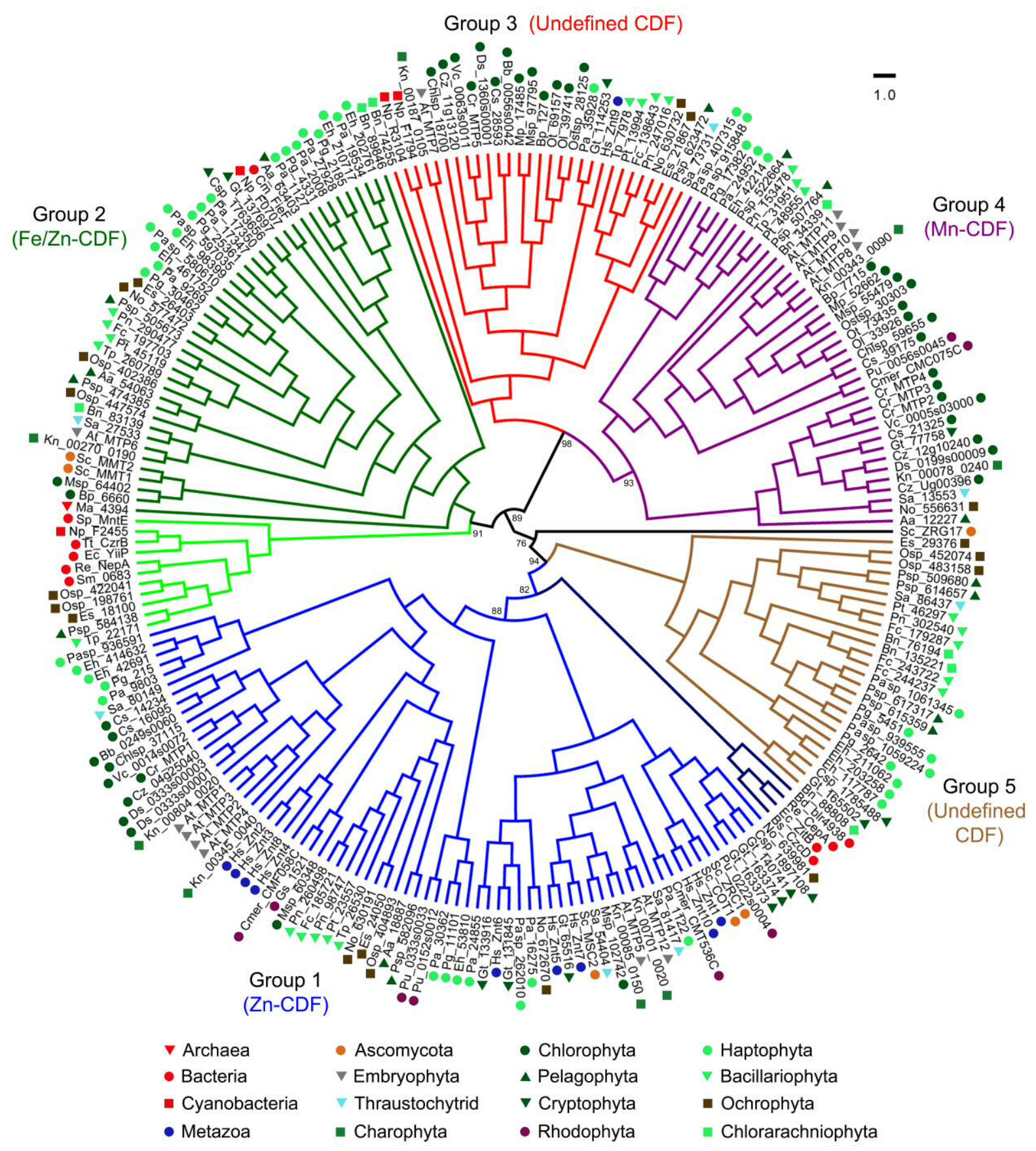

Fig. 1. Phylogenetic analysis of CDF transporter family proteins from various selected algal taxa in comparison to prokaryotic, animal $(H$. sapiens), land plant $(A$. thaliana) and yeast $(S$. cerevisiae) proteins. The genome identifier or accession numbers of all sequences used are provided in Table S1. Coloured symbols indicate the major taxonomic domains of the species where the protein sequence is derived. Five major groups are highlighted. The tree was constructed using the maximum likelihood method and derived from alignments of full length amino acid sequence. A consensus tree following 1000 bootstrap replications is shown, with bootstrap percentage values indicated at the nodes of major branches. The branch length scale bar indicates the evolutionary distance of one amino acid substitution per site. 


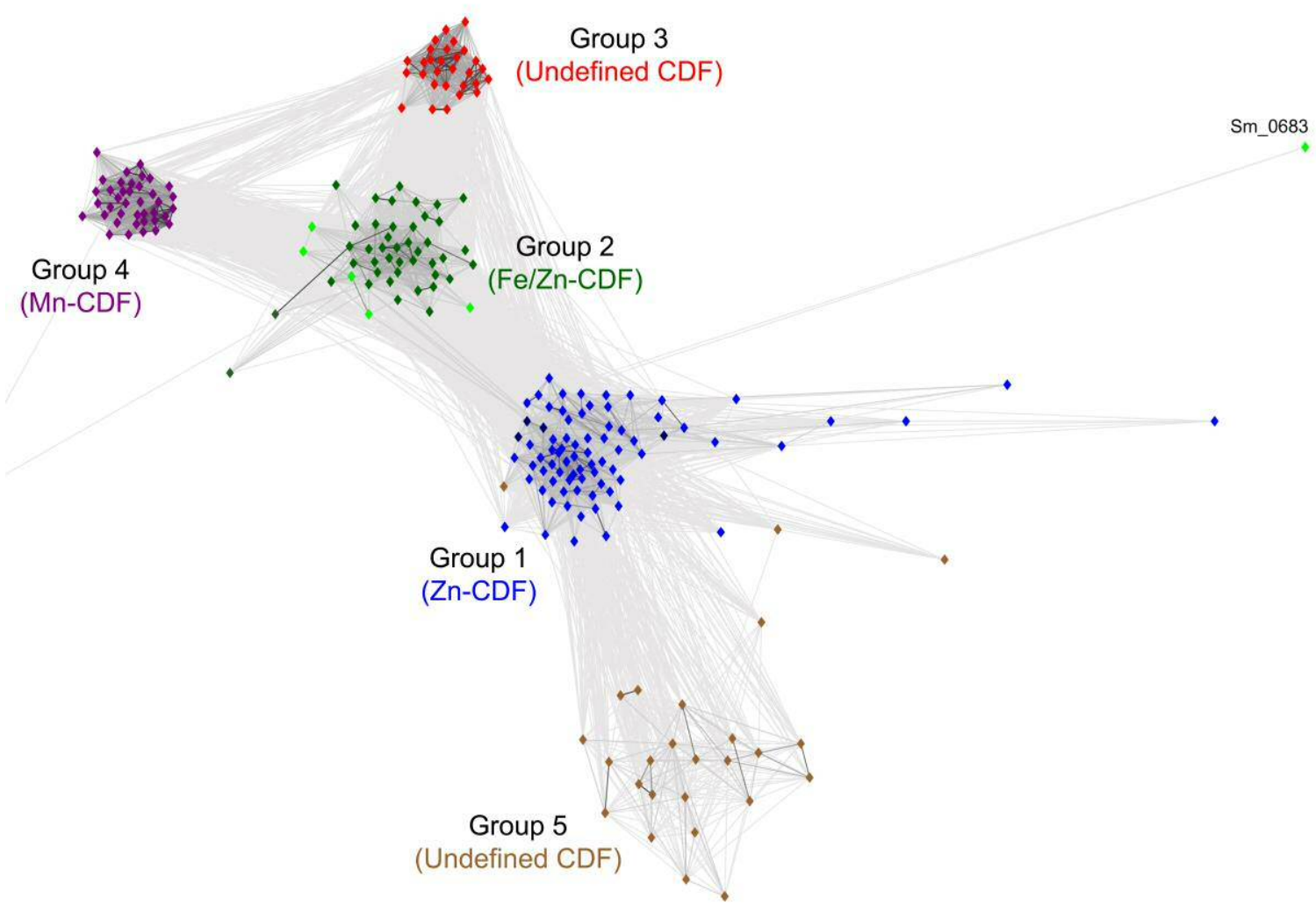

Fig. 2. A two-dimensional cluster analysis of sequences representing the phenetic sequence relationship of CDF transporter family proteins from various selected algal taxa in comparison to prokaryotic, animal $(H$. sapiens), land plant $(A$. thaliana) and yeast ( $S$. cerevisiae) proteins. Each symbol represents a full length amino acid sequence of an individual CDF protein and is coloured on the basis of the phylogenetic groups shown in Fig. 1. Grey lines represent protein connections with reciprocal BLAST hits at $P<10^{-5}$. Six proteins are not shown (five Group 2 members: Psp_584138, Tp_22171, Es_18100, Osp_198761, Osp_422041, and unassigned ScZRG17) as they did not cluster with any of the other sequences and are on the periphery of the plot off the scale. 

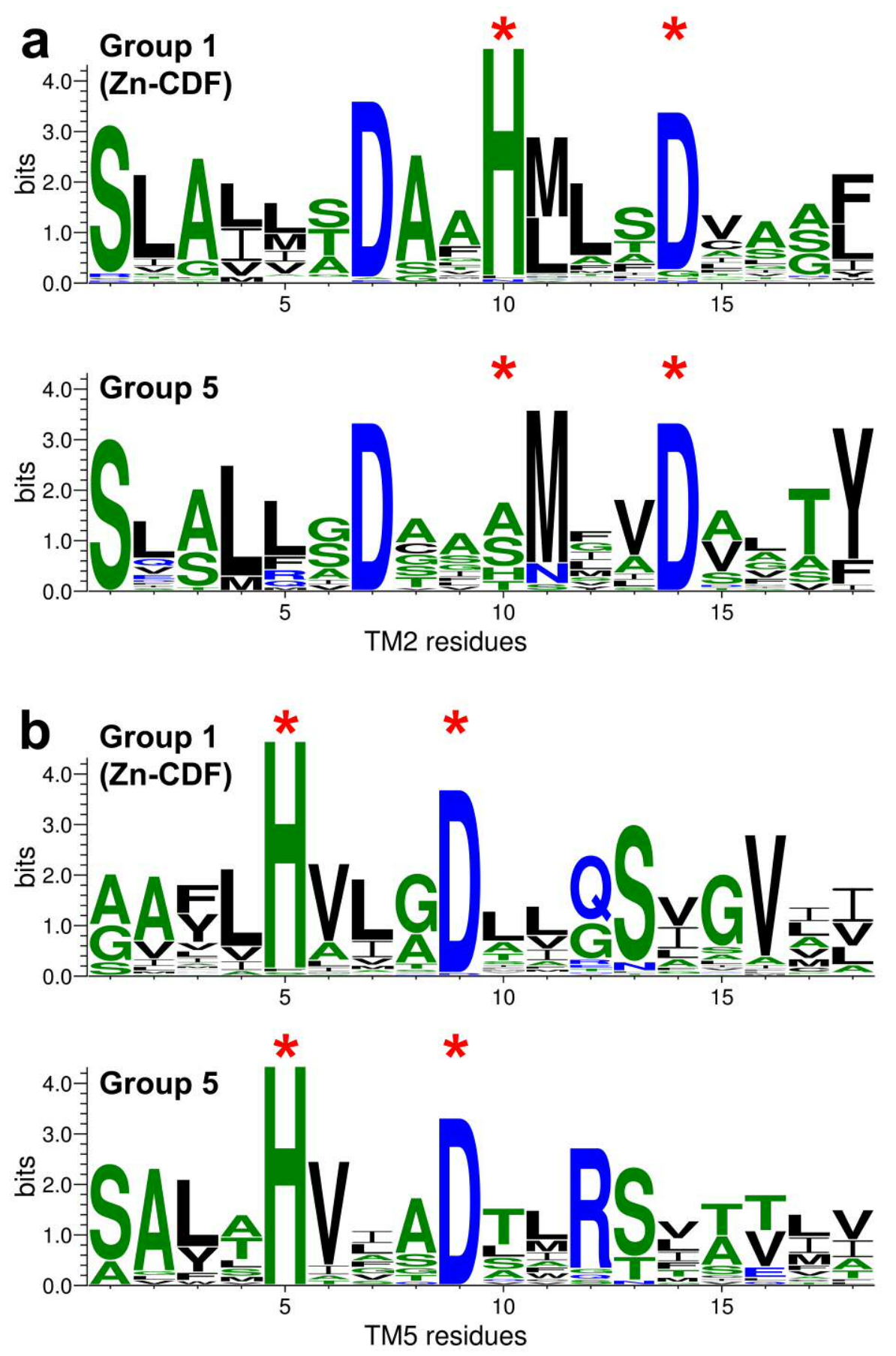

Fig. 3. Comparison of conserved CDF residues within transmembrane (TM) domain 2 (a) and TM domain 5 (b) for Group 1 (Zn-CDF) and Group 5 proteins. Sequence logo representations of 18 residues within each TM domain determined for all of the Group 1 sequences and all of the Group 5 sequences. Asterisks indicate the positions of conserved active site 'A' residues. 

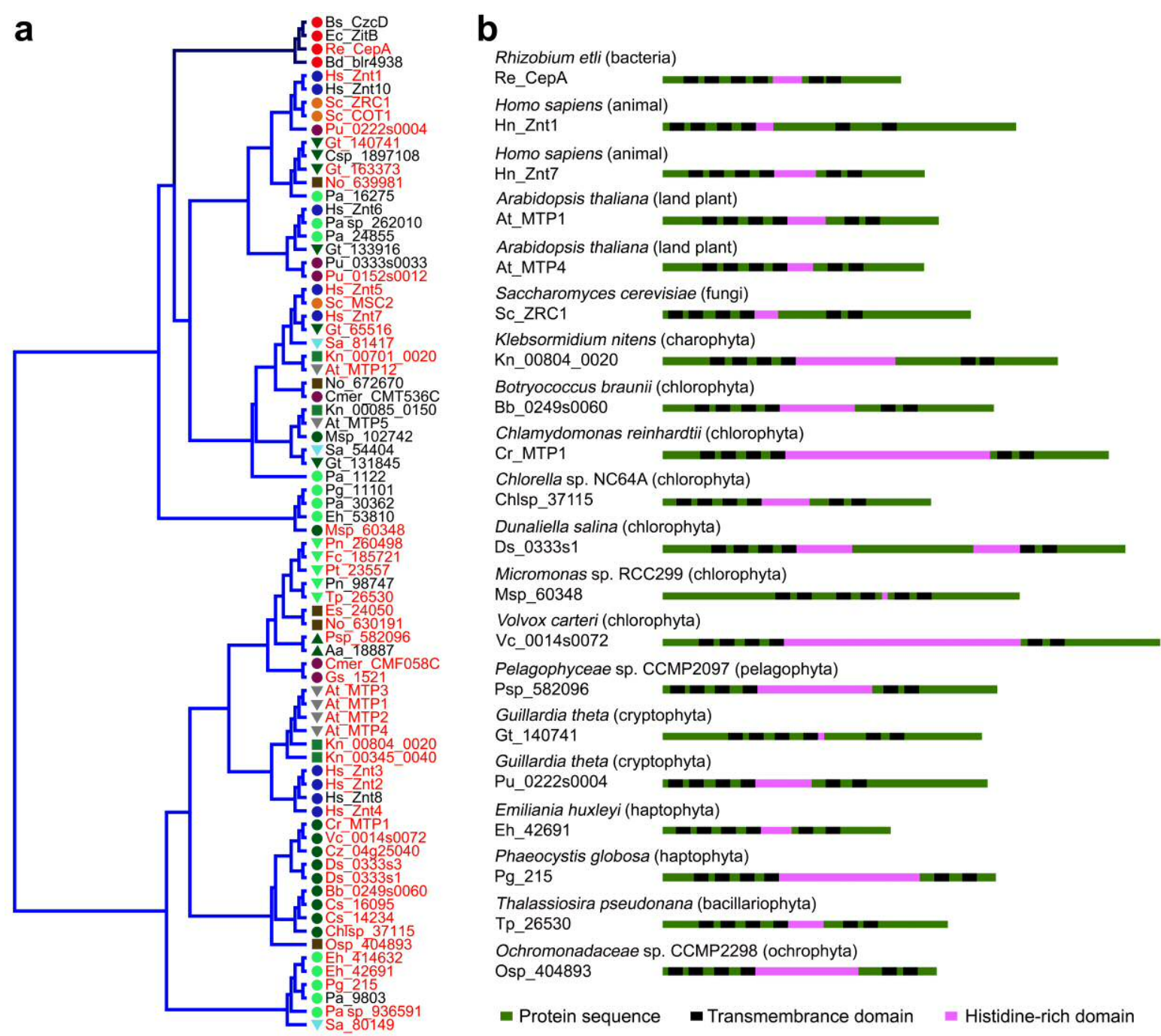

Fig. 4. Analysis of Group $1 \mathrm{Zn}-\mathrm{CDF}$ proteins. (a) Phylogenetic analysis of Zn-CDF family proteins from various selected species. The coloured symbols indicating the major taxonomic domains of each species are as defined in Fig. 1. Proteins highlighted in red possess an obvious histidine-rich domain. (b) Topology models of selected Group $1 \mathrm{Zn}$-CDF proteins indicating the predicted transmembrane (TM) spans and the histidine-rich domain within the major cytosolic loop between TM4 and TM5. 


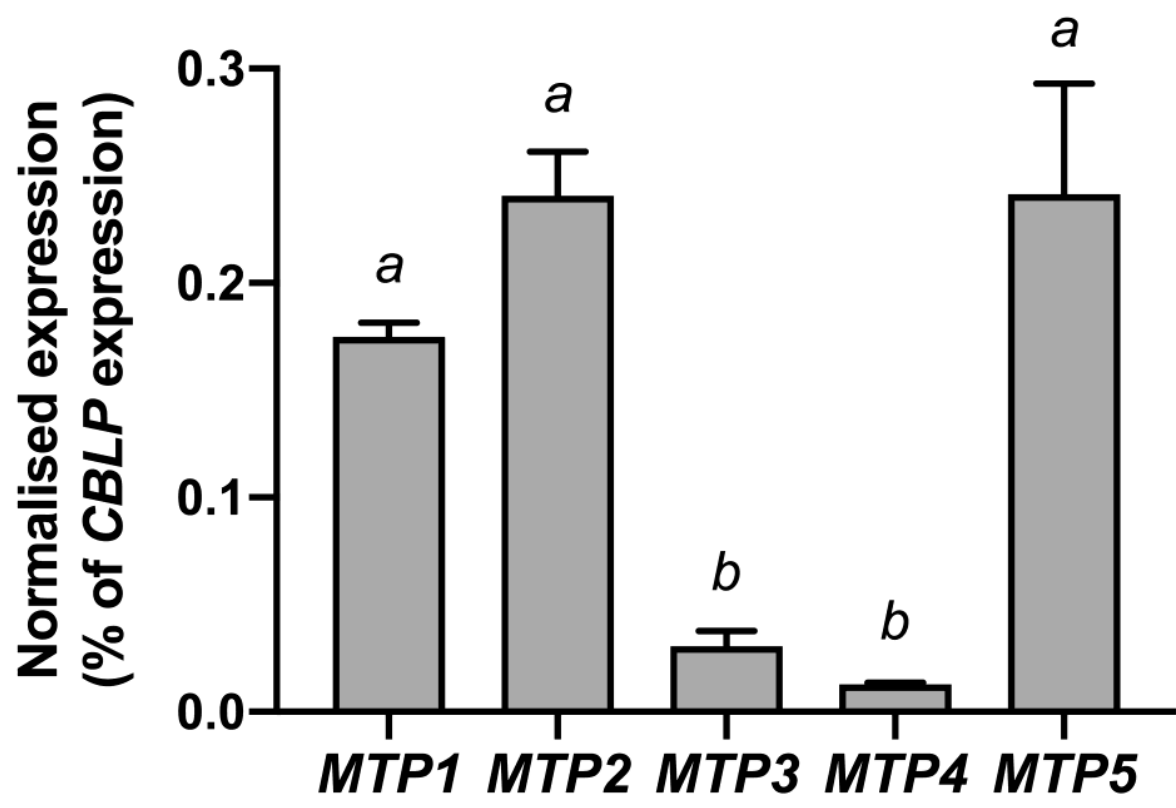

Fig. 5. Expression the CrMTP family genes in $C$. reinhardtii cells grown in standard TAP medium at exponential growth stage. Expression of the selected mRNA transcripts as determined by real-time PCR was calculated as a percentage of CBLP expression. Data points are means $( \pm S E M)$ calculated from biological triplicates. Bars sharing the same lowercase letter indicate no significant difference between treatments $(P>0.05)$ as determined by one-way ANOVA and Tukey post-hoc test. 

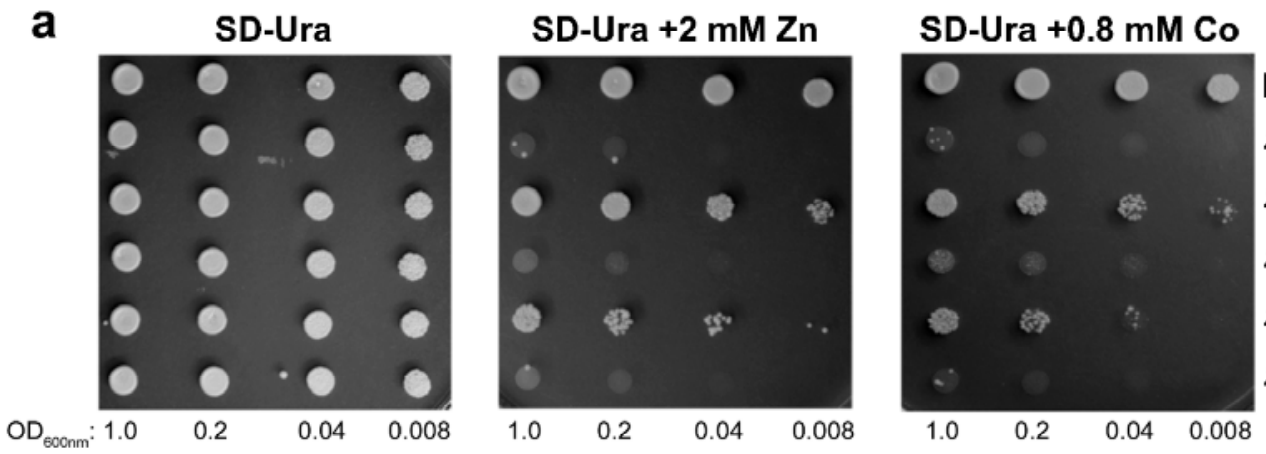

BY4741 (WT)
zrc1cot1 piUGpd
zrc1cot1 MTP1
zrc1cot1 MTP2
zrc1cot1 MTP3
zrc1cot1 MTP4

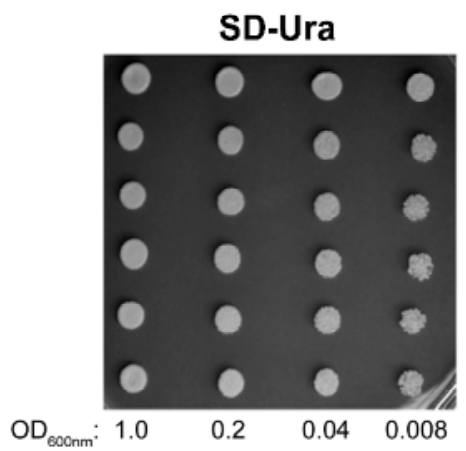

\section{SD-Ura +5 mM Mn}

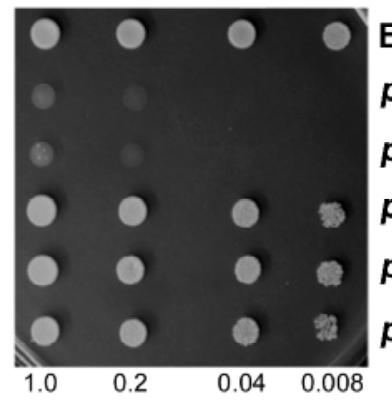

\section{BY4741 (WT) \\ pmr1 piUGpd \\ pmr1 MTP1 \\ pmr1 MTP2 \\ pmr1 MTP3 \\ pmr1 MTP4}

b

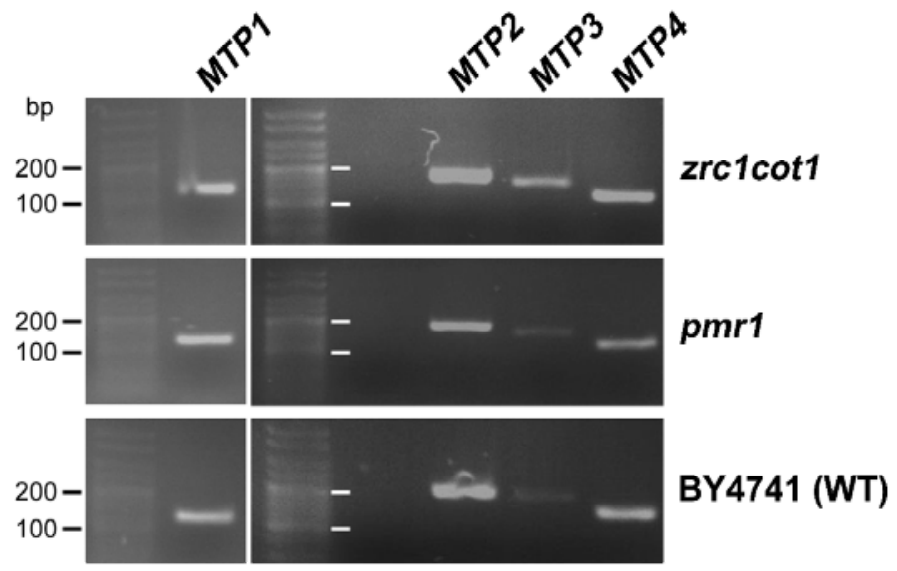

Fig. 6. Heterologous expression of CrMTP1, CrMTP2, CrMTP3 and CrMTP4 in yeast strains. (a) Suppression of Zn, Co and Mn hypersensitivity of the Zn- and Co-sensitive zrc1cot1 yeast mutant and the Mn-sensitive pmr1 mutant by heterologous expression of CrMTP cDNA in comparison with empty vector (piUGpd) and WT (BY4741). Saturated liquid cultures of yeast strains were serially diluted to the indicated cell densities then spotted onto SD -Ura selection medium with or without added Zn, Co or Mn. Yeast growth is shown after 3 d. A representative experiment is shown. (b) RT-PCR of MTP1, MTP2, MTP3 and MTP4 expression in each yeast strain. 
a

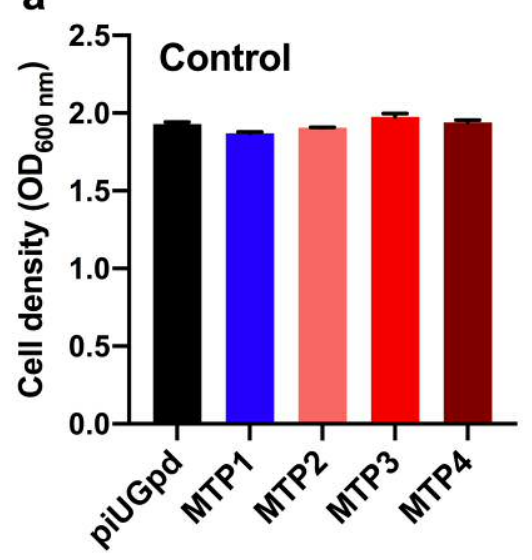

C

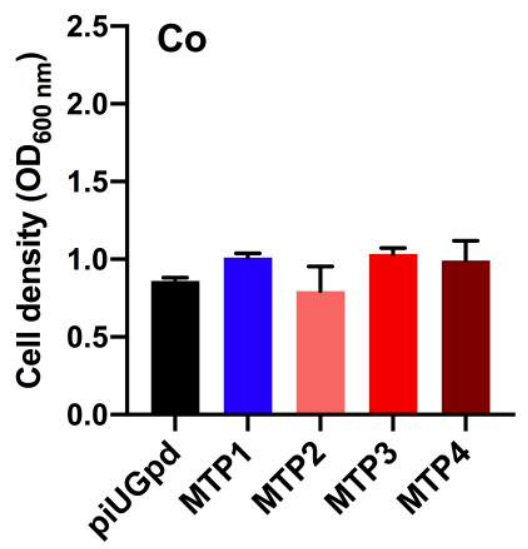

b

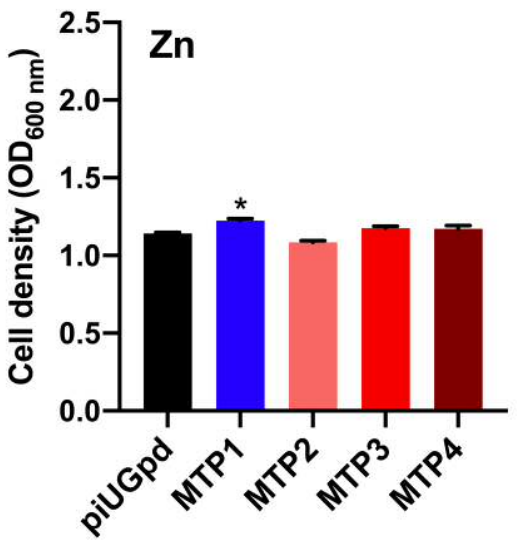

d

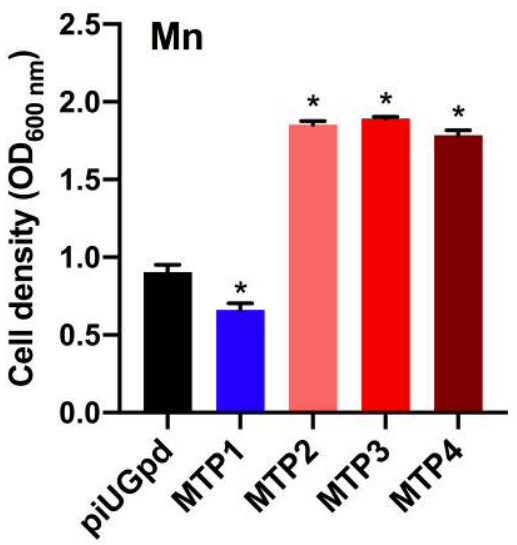

Fig. 7. Heterologous expression of CrMTP1, CrMTP2, CrMTP3 and CrMTP4 in wild type (BY4741) yeast. Cell density measurement of strains expressing each MTP cDNA in comparison with empty vector (piUGpd) grown after $3 \mathrm{~d}$ in liquid SD -Ura medium either without metal exposure (a) or with $10 \mathrm{mM} \mathrm{Zn} \mathrm{(b),} 10 \mathrm{mM}$ Co (c), or $20 \mathrm{mM}$ Mn (d). Data points are means $( \pm$ SEM) from triplicate samples. Bars with an asterisk indicate significant difference between the empty vector control (piUGpd) strain $(P<0.05)$ as determined by one-way ANOVA and Tukey post-hoc test. 

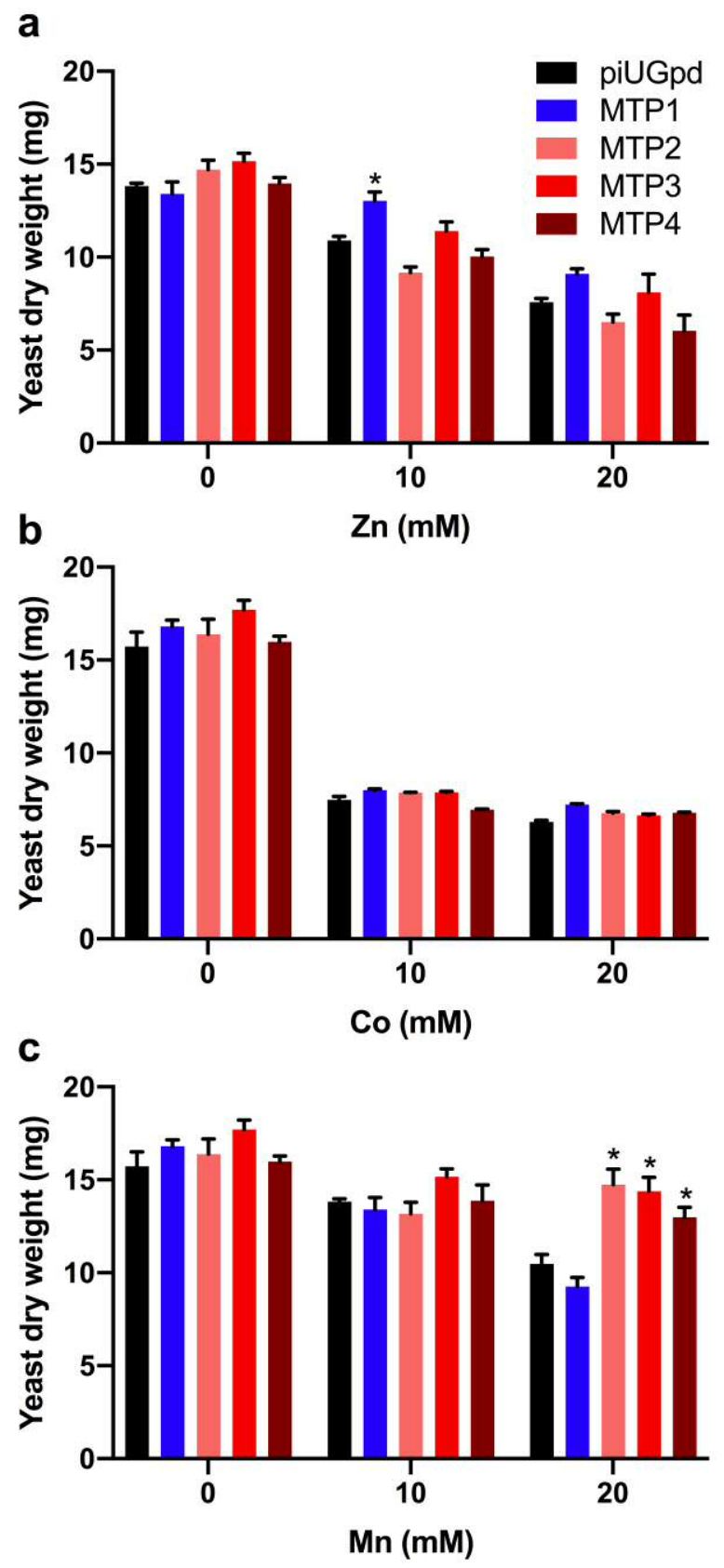

Fig. 8. Heterologous expression of CrMTP1, CrMTP2, CrMTP3 and CrMTP4 in wild type (BY4741) yeast. Dry weight biomass measurement of strains expressing each CrMTP cDNA in comparison with empty vector (piUGpd) grown after $3 \mathrm{~d}$ in liquid SD -Ura medium either without metal exposure or with added Zn (a), Co (b), or Mn (c). Data points are means $( \pm$ SEM) from triplicate samples. Bars with an asterisk indicate significant difference between the empty vector control (piUGpd) strain $(\mathrm{P}<0.05)$ as determined by one-way ANOVA and Tukey post-hoc test. 

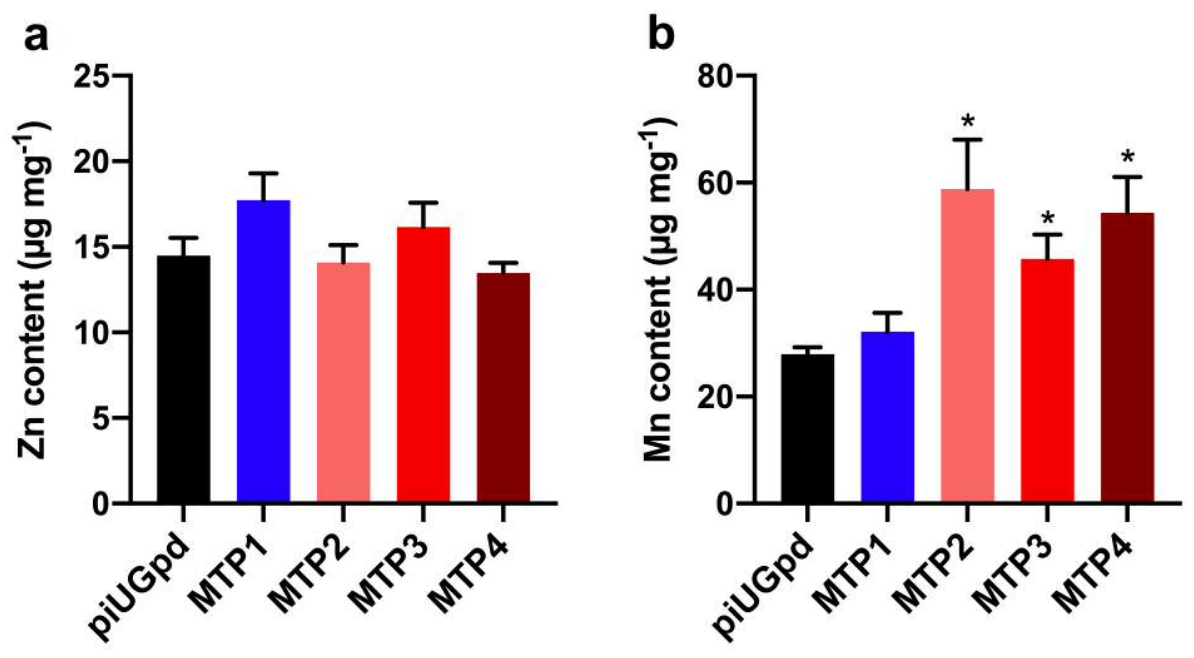

Fig. 9. Zn and Mn uptake into wild type (BY4741) yeast expressing CrMTP1, CrMTP2, CrMTP3 and CrMTP4. Metal content measurement of strains expressing each CrMTP cDNA in comparison with empty vector (piUGpd) grown after $3 \mathrm{~d}$ in liquid SD -Ura medium with added $20 \mathrm{mM} \mathrm{Zn} \mathrm{(a)} \mathrm{or} 20 \mathrm{mM} \mathrm{Mn} \mathrm{(b).} \mathrm{Data} \mathrm{points} \mathrm{are} \mathrm{means} \mathrm{(} \pm$ SEM) from triplicate samples. Bars with an asterisk indicate significant difference between the empty vector control (piUGpd) strain $(P<0.05)$ as determined by one-way ANOVA and Tukey post-hoc test. 